\title{
FLEXURAL BUCKLING DESIGN OF FABRICATED AUSTENITIC AND DUPLEX STAINLESS STEEL COLUMNS
}

\author{
Yang-Lu ${ }^{1, *}$, Zhao-Menghan ${ }^{1}$, Chan-Takming ${ }^{2}$ and Shang-Fan ${ }^{1}$ \\ ${ }^{1}$ The College of Architecture and Civil Engineering; \\ Beijing University of Technology, Beijing 100124, China \\ ${ }^{2}$ Department of Civil and Environmental Engineering, \\ The Hong Kong Polytechnic University, Hung Hom, Kowloon, Hong Kong SAR \\ *(Corresponding author: E-mail: lyang@bjut.edu.cn) \\ Received: 29 September 2016; Revised: 13 February 2017; Accepted: 16 April 2017
}

\begin{abstract}
This paper presents a numerical investigation on the flexural buckling design of fabricated austenitic and duplex stainless steel columns. Complementary experimental investigation was conducted and the numerical modelling methodology was validated against the complementary experimental database. The target parametric study was carried out to assess the influence of initial geometric imperfections, residual stress patterns, mechanical properties, local cross-section slenderness and global slenderness on the structural behaviour. A total of 968 FEA results were developed which included 301 welded box-section and 667 welded I-section columns. The resulting structural performance data were collated to assess the reliability of the current design provisions - EN 1993-1-4 and ASCE 8-02. Results indicate the conservatism on current practices. Improved design provisions were proposed based on the Direct Strength Method, ASCE 8-02 and Perry formulae.
\end{abstract}

Keywords: Flexural buckling design, parametric analysis, reliability analysis, stainless steel, columns

DOI: 10.18057/IJASC.2018.14.2.4

\section{INTRODUCTION}

In recent years, stainless steel has been widely used for construction because of their favorable material properties and the corrosion resistance [1]. Corresponding stainless steel design specifications have also been developed and adopted in Europe [2], the United States [3] and China [4] which promotes the further acceptance by the engineers. These days, research on stainless steel focuses on material characterization, residual stress patterns, local and global stability behaviours. Over the research on the material characterization, Mirambell and Real [5] proposed a two-stage Ramberg-Osgood model which was subsequently modified by Quach [6] to a three-stage model. Research on residual stress measurements were conducted by Gardner [7], Quach [8] and Yuan [9]. Wang [10] and Yang [11], on the other hand, developed an overall stability calculation method for welded I-section stainless steel beams through experimental and theoretical investigations. Yuan [12] studied the local-overall interaction buckling of welded stainless steel columns experimentally and proposed a new design method. Research on stability loading capacity of cold-formed stainless steel columns was also carried out by Shu $[13,14]$. Two modified design methods for lean duplex stainless steel beam-columns was also proposed in Huang and Young [15]. A new design method Continuous strength method, which can further exploit the strain-hardening benefits of the stainless steel materials, has been developed in the last two decades by Gardner and Nethercot [16-18].

Current design specifications for stainless steel include EN 1993-1-4 (for cold-formed and welded members), SEI/ASCE 8-02 and AS/NZS 4673-2001 (for cold-formed members) [19]. The design methodologies in EN 1993-1-4 and AS/NZS 4673-2001 are fundamentally the same as their carbon steel design counterparts. 
To expand the current stainless steel research to welded/fabricated sections, the objective of this paper is to develop an efficient method for flexural buckling design on fabricated austenitic and duplex stainless steel columns. Experimental programme, followed by the numerical investigation, was discussed in two companion papers [20,21]. Experimental investigation on twenty-two austenitic and duplex stainless steel welded I-section compression members were conducted by Yang et al. [20] while twelve austenitic and duplex welded stainless steel box-section columns were tested by Yang et al. [21]. A corresponding numerical model was developed to replicate the experimental procedure. The load-displacement curves and buckling strength of experiments and finite element simulations were compared, indicating that the finite element methodology is applicable for parametric studies.

Considering the conservatism of existing codified approaches during the stage prior to the yield stress, the focus of the present paper was the development of new efficient methods, i.e. a three-segment model, for the design of welded stainless steel cross-sections under compressive loading, based on the experimental results from the companion papers and parametric studies conducted herein. The reliability of the proposed design method will be assessed by comparing the design proposal with generated structural performance data.

\section{NUMERICAL MODELING}

\subsection{Introduction}

In conjunction with the previously conducted experimental studies [20,21], a numerical modeling programme was performed using the general-purpose finite element analysis package ANSYS. The aims of the numerical investigations were initially to replicate the full experimental load-deformation curves and to assess the sensitivity of the FE models to extensive input parameters, and subsequently to conduct parametric studies to generate further structural performance data to supplement the experimental results. The accuracy of the FE models was assessed in the companion paper $[20,21]$ by comparing the key results, full load-deformation histories and failure modes obtained from tests with those derived from the numerical simulations.

\subsection{Parametric Analysis}

Having verified the general ability of the FE models to replicate the column test behaviour, a series of parametric studies were conducted. The basic FE model took $0.001 L$ as initial geometric imperfection factor, where $L$ is the length of the member. Residual stress distribution model proposed by Yuan Huanxin [9], the measured material properties and geometric dimensions were used as based modelling parameters. The primary aim of the parametric studies was to investigate the influence of initial global geometric imperfection, residual stress, material properties, local width-to-thickness ratio and non-dimensional global slenderness on the column load carrying capacity. The obtained results were also used to assess column buckling design curves.

\subsection{Global Geometric Imperfection}

Three different amplitudes of the global geometric imperfection, including $0.0005 \mathrm{~L}, 0.001 \mathrm{~L}$, and $0.002 L$, in the shape of a half-sine wave, were used. Table 1 and Table 2 show the FE results of column loading capacity with different geometric imperfections. The comparison of buckling resistance by using different amplitudes is also presented in Figure 1. It illustrates that: 
Table 1. FE Results with Different Geometric Imperfections (Box-section)

\begin{tabular}{cccccccccccc}
\hline \multicolumn{1}{c}{ uustenitic stainless steel } \\
\hline $\bar{\lambda}$ & $\begin{array}{c}F_{0.001} \\
(\mathrm{kN})\end{array}$ & $\begin{array}{c}F_{0.002} \\
(\mathrm{kN})\end{array}$ & $\begin{array}{c}F_{0.0005} \\
(\mathrm{kN})\end{array}$ & $\begin{array}{c}F_{0.002} / \\
F_{0.001}\end{array}$ & $\begin{array}{c}F_{0.0005} / \\
F_{0.001}\end{array}$ & $\bar{\lambda}$ & $\begin{array}{c}F_{0.001} \\
(\mathrm{kN})\end{array}$ & $\begin{array}{c}F_{0.002} \\
(\mathrm{kN})\end{array}$ & $\begin{array}{c}F_{0.0005} \\
(\mathrm{kN})\end{array}$ & $\begin{array}{c}F_{0.002} / F_{0.001} \\
F_{0.0005} / \\
F_{0.001}\end{array}$ \\
\hline 0.49 & 771 & 739 & 795 & 0.96 & 1.03 & 0.74 & 1362 & 1287 & 1402 & 0.94 & 1.03 \\
0.74 & 627 & 589 & 653 & 0.94 & 1.04 & 0.94 & 1132 & 1054 & 1176 & 0.93 & 1.04 \\
0.99 & 491 & 457 & 515 & 0.93 & 1.04 & 1.15 & 907 & 842 & 946 & 0.93 & 1.04 \\
1.24 & 378 & 348 & 398 & 0.92 & 1.05 & 1.35 & 722 & 670 & 748 & 0.93 & 1.04 \\
1.48 & 290 & 266 & 308 & 0.92 & 1.06 & 1.55 & 576 & 537 & 597 & 0.93 & 1.04 \\
1.73 & 225 & 207 & 239 & 0.92 & 1.06 & 1.75 & 466 & 436 & 482 & 0.93 & 1.03 \\
1.98 & 178 & 164 & 188 & 0.92 & 1.06 & 1.96 & 382 & 359 & 395 & 0.94 & 1.03 \\
2.23 & 143 & 132 & 151 & 0.92 & 1.06 & 2.16 & 318 & 300 & 329 & 0.94 & 1.03 \\
2.47 & 117 & 109 & 124 & 0.93 & 1.05 & 2.36 & 269 & 254 & 278 & 0.94 & 1.03 \\
\hline
\end{tabular}

Table 2. FE Results with Different Geometric Imperfections (I-section)

\begin{tabular}{|c|c|c|c|c|c|c|c|c|c|c|c|c|}
\hline & \multicolumn{6}{|c|}{ Austenitic stainless steel } & \multicolumn{6}{|c|}{ Duplex stainless steel } \\
\hline & $\bar{\lambda}$ & $\begin{array}{l}F_{0.001} \\
(\mathrm{kN})\end{array}$ & $\begin{array}{c}F_{0.0005} \\
(\mathrm{kN})\end{array}$ & $\begin{array}{l}F_{0.002} \\
(\mathrm{kN})\end{array}$ & $\begin{array}{c}F_{0.0005} / \\
F_{0.001}\end{array}$ & $\begin{array}{l}F_{0.002} / \\
F_{0.001}\end{array}$ & $\bar{\lambda}$ & $\begin{array}{l}F_{0.001} \\
(\mathrm{kN})\end{array}$ & $\begin{array}{c}F_{0.0005} \\
(\mathrm{kN})\end{array}$ & $\begin{array}{l}F_{0.002} \\
(\mathrm{kN})\end{array}$ & $\begin{array}{c}F_{0.0005} / \\
F_{0.001} \\
\end{array}$ & $\begin{array}{l}F_{0.002 /} \\
F_{0.001} \\
\end{array}$ \\
\hline \multirow{9}{*}{$\begin{array}{l}\text { Major } \\
\text { axis }\end{array}$} & 0.39 & 905 & 937 & 855 & 1.04 & 0.94 & 0.50 & 1763 & 1830 & 1672 & 1.04 & 0.95 \\
\hline & 0.59 & 764 & 788 & 713 & 1.03 & 0.93 & 0.71 & 1471 & 1530 & 1380 & 1.04 & 0.94 \\
\hline & 0.79 & 640 & 663 & 586 & 1.04 & 0.92 & 0.92 & 1182 & 1240 & 1103 & 1.05 & 0.93 \\
\hline & 0.99 & 523 & 550 & 478 & 1.05 & 0.92 & 1.13 & 938 & 980 & 874 & 1.04 & 0.93 \\
\hline & 1.18 & 429 & 451 & 390 & 1.05 & 0.91 & 1.34 & 746 & 778 & 697 & 1.04 & 0.93 \\
\hline & 1.38 & 353 & 370 & 321 & 1.05 & 0.91 & 1.56 & 601 & 623 & 564 & 1.04 & 0.94 \\
\hline & 1.58 & 292 & 305 & 266 & 1.04 & 0.91 & 1.77 & 491 & 506 & 463 & 1.03 & 0.94 \\
\hline & 1.77 & 244 & 253 & 223 & 1.04 & 0.92 & 1.98 & 408 & 418 & 386 & 1.03 & 0.94 \\
\hline & 1.97 & 203 & 213 & 189 & 1.05 & 0.93 & 2.19 & 343 & 351 & 326 & 1.02 & 0.95 \\
\hline \multirow{10}{*}{$\begin{array}{l}\text { Minor } \\
\text { axis }\end{array}$} & 0.53 & 923 & 942 & 884 & 1.02 & 0.96 & 0.66 & 1795 & 1866 & 1707 & 1.04 & 0.95 \\
\hline & 0.73 & 759 & 780 & 711 & 1.03 & 0.94 & 0.84 & 1539 & 1604 & 1443 & 1.04 & 0.94 \\
\hline & 0.92 & 611 & 636 & 566 & 1.04 & 0.93 & 1.01 & 1292 & 1359 & 1199 & 1.05 & 0.93 \\
\hline & 1.12 & 487 & 511 & 450 & 1.05 & 0.92 & 1.19 & 1064 & 1120 & 986 & 1.05 & 0.93 \\
\hline & 1.32 & 394 & 416 & 362 & 1.06 & 0.92 & 1.37 & 874 & 918 & 811 & 1.05 & 0.93 \\
\hline & 1.52 & 323 & 342 & 297 & 1.06 & 0.92 & 1.54 & 722 & 760 & 672 & 1.054 & 0.93 \\
\hline & 1.72 & 269 & 285 & 247 & 1.06 & 0.92 & 1.72 & 605 & 635 & 563 & 1.05 & 0.93 \\
\hline & 1.91 & 228 & 240 & 208 & 1.05 & 0.91 & 1.90 & 511 & 534 & 476 & 1.04 & 0.93 \\
\hline & 2.11 & 194 & 204 & 178 & 1.05 & 0.92 & 2.07 & 436 & 455 & 408 & 1.04 & 0.93 \\
\hline & 2.31 & 168 & 175 & 154 & 1.05 & 0.92 & 2.25 & 376 & 391 & 352 & 1.04 & 0.94 \\
\hline
\end{tabular}




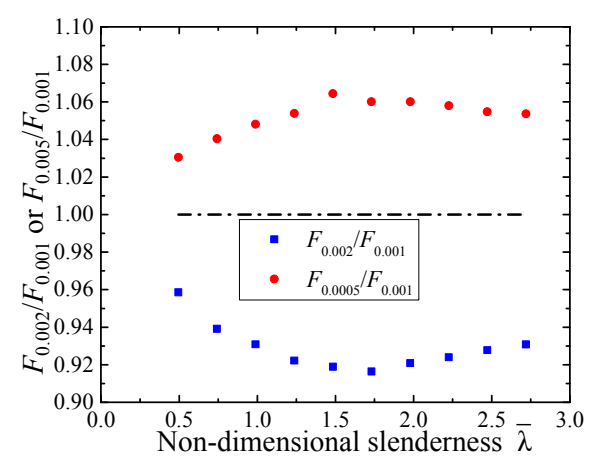

(a) Austenitic box-section

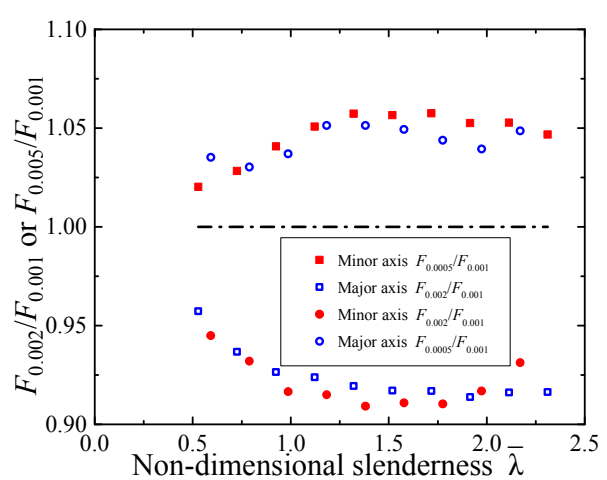

(c) Austenitic I-section

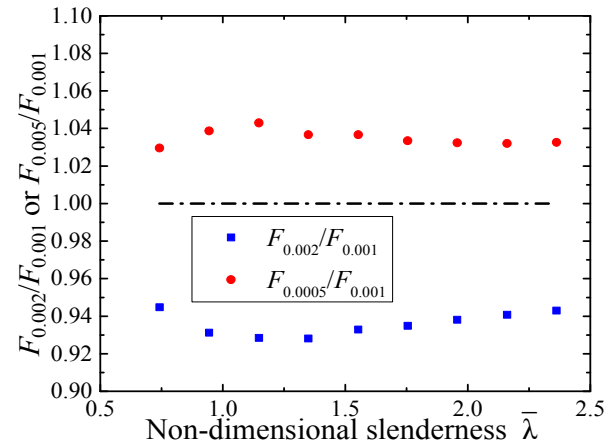

(b) Duplex box-section

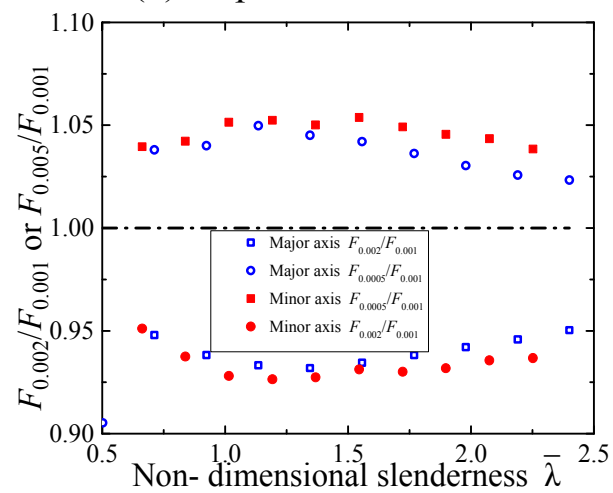

(d) Duplex I-section

Figure 1. The Influence of Initial Imperfection

o The influence of column geometric imperfections on the loading capacity is related to column slenderness. Columns with medium lengths, as anticipated, are more sensitive to different geometric imperfections;

o $F_{0.0005}$ is generally $5 \%$ higher than $F_{0.001}$, while $F_{0.001}$ is generally $7 \%$ higher than $F_{0.002}$. And the ultimate load is slightly fluctuated with the change of non-dimensional slenderness ratio;

o For columns buckling about minor axis, the influence of geometric imperfections on loading capacity is slightly more obvious.

\section{$2.4 \quad$ Residual Stress}

The residual stress measurements were conducted in $[20,21]$ and the corresponding models were proposed as shown in Figure 2. This section compares the FE results with and without the consideration of residual stress and the results are summarized in Table 3 and Table 4 respectively. All data are also compared in Figure 3. In Figure 3, the horizontal axis is the non-dimensional slenderness ratio and the vertical axis is the ratio of ultimate load $F_{\mathrm{w}}$ to $F_{\mathrm{y}} . F_{\mathrm{w}}$ and $F_{\mathrm{y}}$ represent the loading capacity with and without considering the residual stress respectively. It shows that

o The influence of residual stress upon the loading capacity is related to column slenderness. Box-section columns with larger slenderness are more sensitive to residual stress. For columns buckling about major axis, residual stress affects slender columns more than stub counterparts. While for columns buckling about minor axis, residual stress affects medium-length columns the most.

o Columns buckling about minor axis are more sensitive to residual stress.

Therefore, residual stress was considered in all models in the subsequent parametric analysis. 


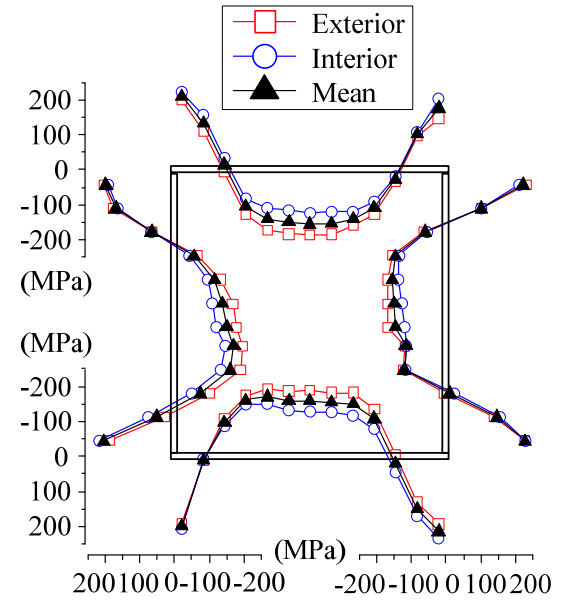

(a) Austenitic box-sections

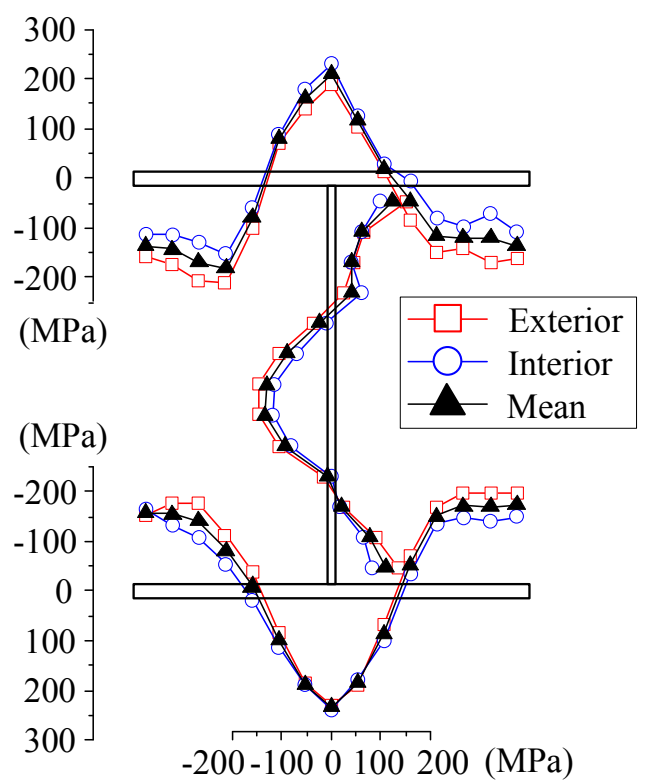

(c) Austenitic I-sections

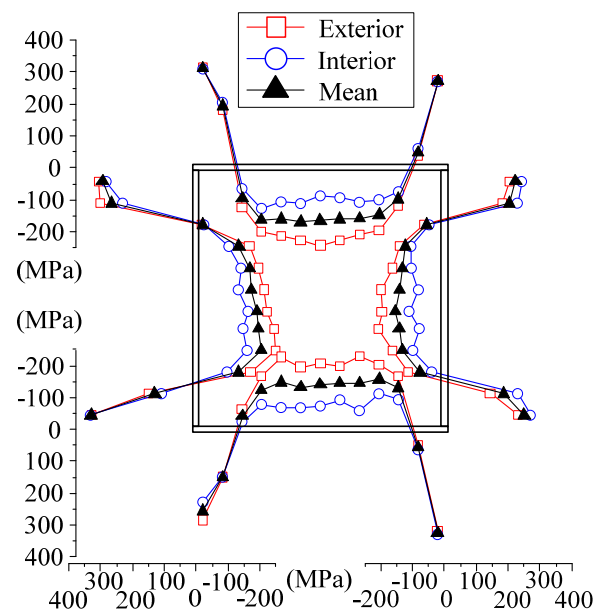

(b) Duplex box-sections

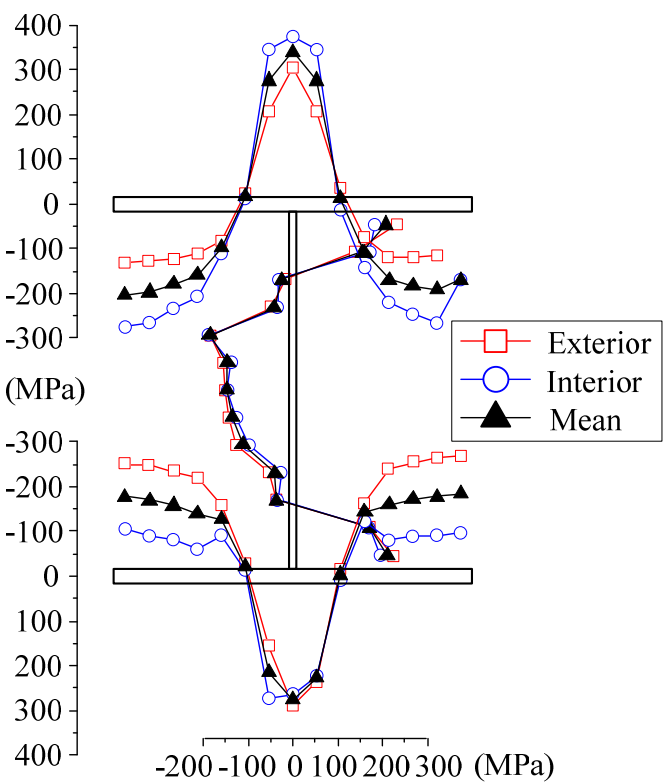

(d) Duplex I-sections

Figure 2. Residual Stress Distribution in [9]

Table 3. FE Results with (Fy) and without (Fw) Residual Stress (Box-section)

\begin{tabular}{cccccccc}
\hline \multicolumn{4}{c}{ Austenitic stainless steel } & \multicolumn{5}{c}{ Duplex stainless steel } \\
\hline $\bar{\lambda}$ & $F_{\mathrm{y}}(\mathrm{kN})$ & $F_{\mathrm{w}}(\mathrm{kN})$ & $F_{\mathrm{w}} / F_{\mathrm{y}}$ & $\bar{\lambda}$ & $F_{\mathrm{y}}(\mathrm{kN})$ & $F_{\mathrm{w}}(\mathrm{kN})$ & $F_{\mathrm{w}} / F_{\mathrm{y}}$ \\
\hline 0.49 & 771 & 759 & 0.98 & 0.74 & 1564 & 1561 & 1.00 \\
0.74 & 627 & 618 & 0.98 & 0.94 & 1362 & 1362 & 1.00 \\
0.99 & 491 & 494 & 1.01 & 1.15 & 1132 & 1139 & 1.01 \\
1.24 & 378 & 390 & 1.03 & 1.35 & 907 & 922 & 1.02 \\
1.48 & 289 & 307 & 1.06 & 1.55 & 722 & 738 & 1.02 \\
1.73 & 225 & 242 & 1.07 & 1.75 & 575 & 594 & 1.03 \\
1.98 & 177 & 193 & 1.09 & 1.96 & 466 & 484 & 1.04 \\
2.22 & 143 & 157 & 1.10 & 2.14 & 382 & 399 & 1.04 \\
2.47 & 117 & 130 & 1.11 & 2.36 & 318 & 333 & 1.05 \\
\hline
\end{tabular}


Table 4. FE Results with $(F \mathrm{y})$ and without $\left(F_{\mathrm{W}}\right)$ Residual Stress (I-section)

\begin{tabular}{ccccccccc}
\hline & \multicolumn{3}{c}{ Austenitic stainless steel } & \multicolumn{5}{c}{ Duplex stainless steel } \\
\hline \multirow{7}{*}{$\bar{\lambda}$} & $F_{\mathrm{w}}(\mathrm{kN})$ & $F_{\mathrm{y}}(\mathrm{kN})$ & $F_{\mathrm{w}} / F_{\mathrm{y}}$ & $\bar{\lambda}$ & $F_{\mathrm{w}}(\mathrm{kN})$ & $F_{\mathrm{y}}(\mathrm{kN})$ & $F_{\mathrm{w}} / F_{\mathrm{y}}$ \\
\hline \multirow{4}{*}{ Major } & 0.39 & 1045 & 1044 & 1.00 & 0.50 & 0 & 2280 & 0.00 \\
axis & 0.59 & 899 & 905 & 0.99 & 0.71 & 1779 & 1763 & 1.01 \\
& 0.79 & 760 & 764 & 0.99 & 0.92 & 1482 & 1471 & 1.01 \\
& 1.18 & 537 & 523 & 1.03 & 1.34 & 946 & 938 & 1.01 \\
& 1.38 & 445 & 429 & 1.04 & 1.56 & 758 & 746 & 1.01 \\
& 1.58 & 367 & 352 & 1.04 & 1.77 & 612 & 601 & 1.02 \\
& 1.77 & 305 & 292 & 1.04 & 1.98 & 500 & 491 & 1.02 \\
& 1.97 & 256 & 243 & 1.05 & 2.19 & 414 & 408 & 1.01 \\
& 2.17 & 217 & 203 & 1.07 & 2.40 & 348 & 343 & 1.01 \\
\hline \multirow{4}{*}{ Minor } & 0.53 & 962 & 923 & 1.04 & 0.66 & 1843 & 1795 & 1.03 \\
axis & 0.73 & 820 & 759 & 1.08 & 0.84 & 1582 & 1539 & 1.03 \\
& 1.32 & 460 & 393 & 1.17 & 1.37 & 897 & 874 & 1.02 \\
& 1.52 & 379 & 323 & 1.17 & 1.54 & 740 & 722 & 1.02 \\
& 1.72 & 314 & 269 & 1.16 & 1.72 & 617 & 605 & 1.02 \\
& 1.91 & 262 & 228 & 1.15 & 1.90 & 520 & 511 & 1.02 \\
& 2.11 & 221 & 194 & 1.14 & 2.07 & 443 & 436 & 1.02 \\
& 2.31 & 189 & 168 & 1.12 & 2.25 & 381 & 376 & 1.01 \\
\hline
\end{tabular}

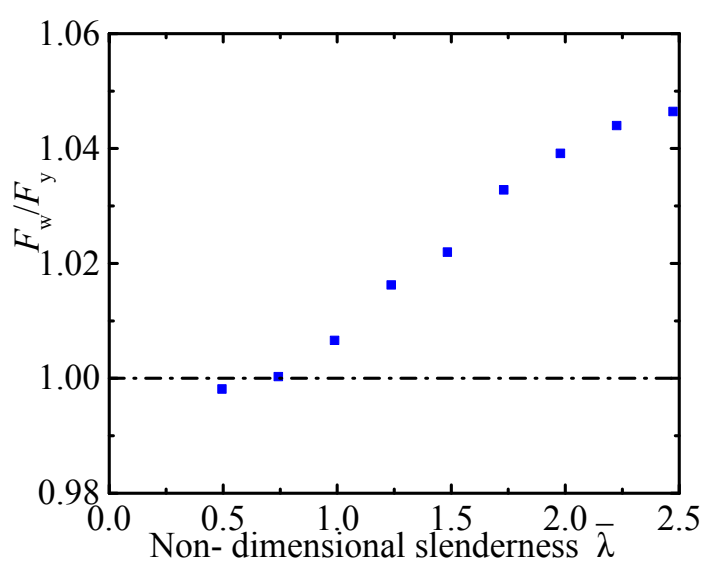

(a) Austenitic box-section

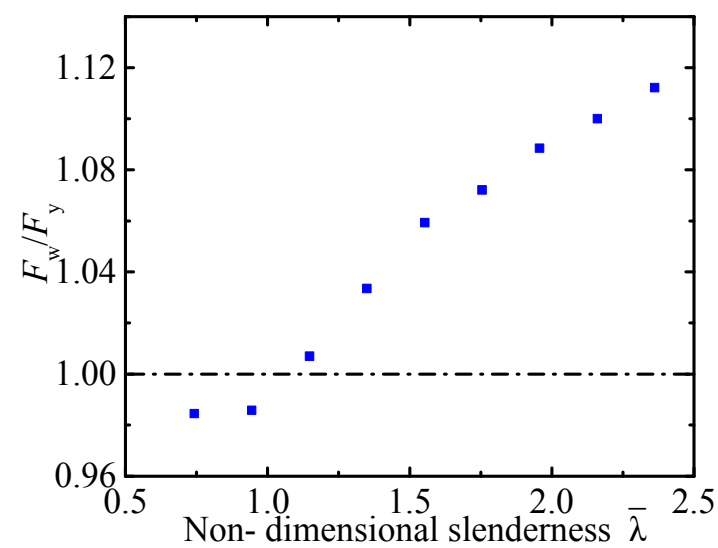

(b) Duplex box-section 


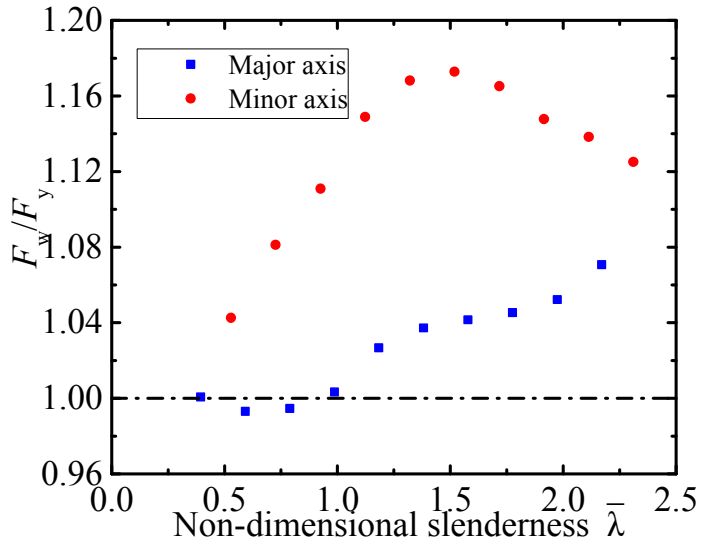

(c) Austenitic I-section

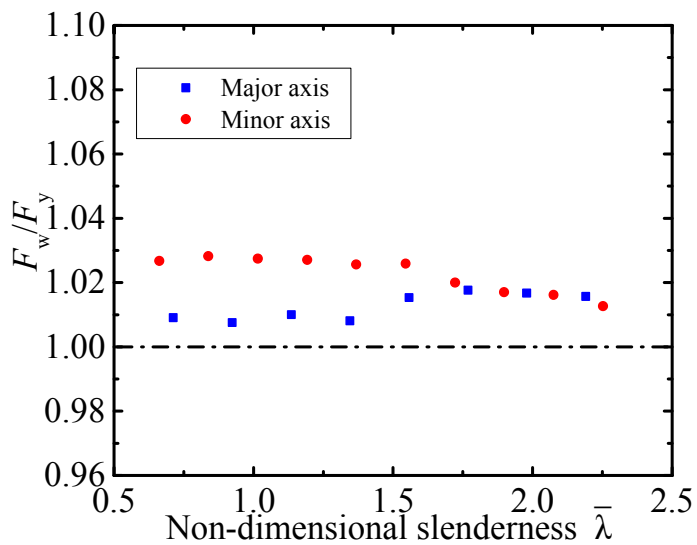

(d) Duplex I-section

Figure 3. The Influence of Residual Stress

\subsection{Material Properties}

Material curves of $6 \mathrm{~mm}$ and $10 \mathrm{~mm}$ stainless steel plates are used for numerical analysis to assess the influence of material properties. The two based material curves are shown in Figure 4. The parametric results are shown in Table 5-6 and Figure 5. In Figure 5, the horizontal axis is the non-dimensional slenderness ratio, the vertical axis is the ratio of ultimate load of two different materials $F_{1}$ (for $6 \mathrm{~mm}$ ), $F_{2}$ (for $10 \mathrm{~mm}$ ). It can be observed that:

0 The influence of material properties on the loading capacity is related to column slenderness, i.e. columns with smaller slenderness are more sensitive to material properties;

o Columns buckling about minor axis are more sensitive to material properties;

o The influence of material properties on duplex stainless steel columns is more obvious than that of austenitic stainless steel.

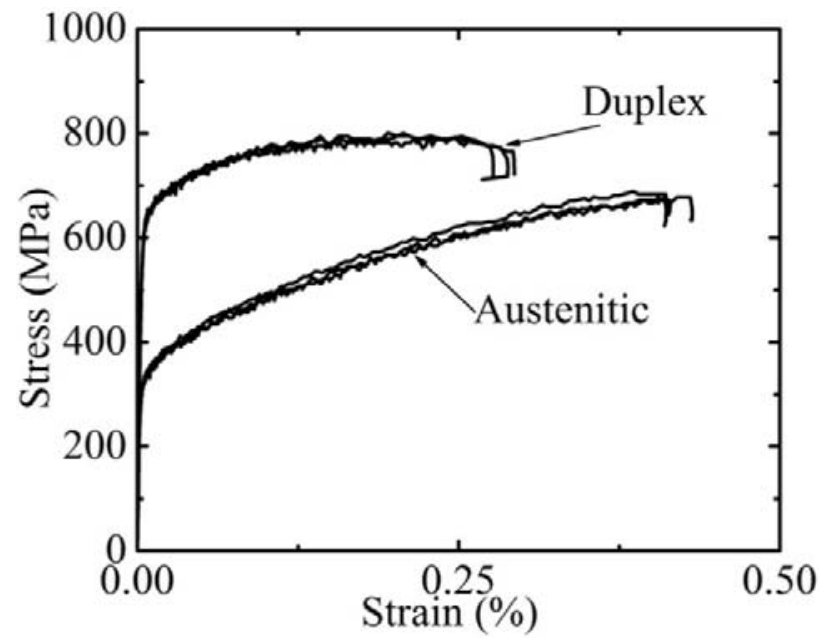

(a) $6 \mathrm{~mm}$ plates 


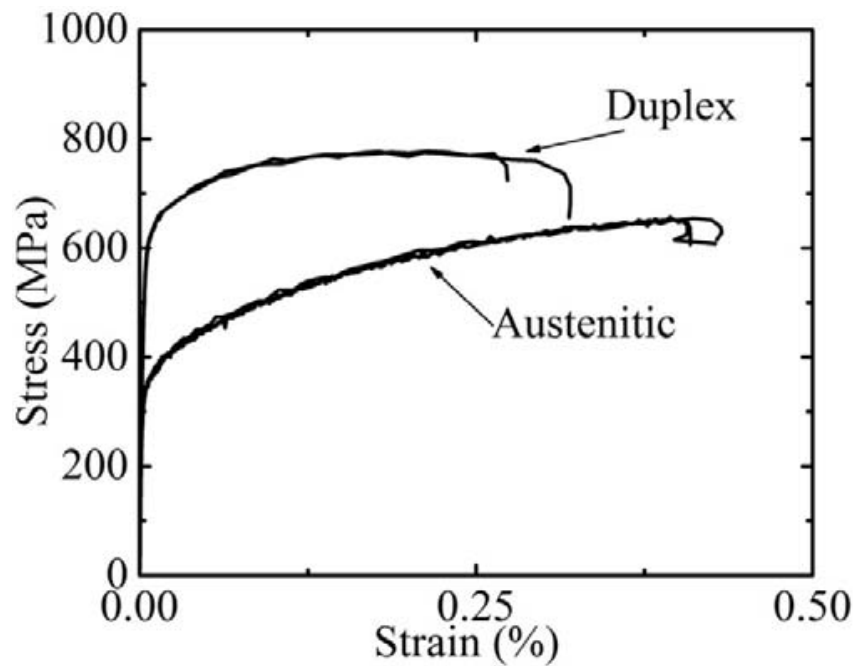

(b) $10 \mathrm{~mm}$ plates

Figure 4. Material Curves of $6 \mathrm{~mm}$ and $10 \mathrm{~mm}$ Plates

Table 5. FE Results with Different Material Properties $-F_{1}$ for $6 \mathrm{~mm}$ and $F_{2}$ for $10 \mathrm{~mm}$ (Box-section)

\begin{tabular}{cccccccc}
\hline \multicolumn{4}{c}{ Austenitic stainless steel } & \multicolumn{5}{c}{ Duplex stainless steel } \\
\hline $\bar{\lambda}$ & $F_{1}(\mathrm{kN})$ & $F_{2}(\mathrm{kN})$ & $F_{2} / F_{1}$ & $\bar{\lambda}$ & $F_{1}(\mathrm{kN})$ & $F_{2}(\mathrm{kN})$ & $F_{2} / F_{1}$ \\
\hline 0.49 & 775 & 813 & 1.05 & 0.54 & 1564 & 1500 & 1.04 \\
0.74 & 638 & 675 & 1.06 & 0.74 & 1362 & 1298 & 1.05 \\
0.99 & 502 & 528 & 1.05 & 0.94 & 1132 & 1079 & 1.05 \\
1.24 & 387 & 401 & 1.03 & 1.15 & 907 & 878 & 1.03 \\
1.48 & 295 & 303 & 1.02 & 1.35 & 722 & 701 & 1.03 \\
1.73 & 229 & 232 & 1.01 & 1.55 & 575 & 565 & 1.02 \\
1.98 & 180 & 182 & 1.01 & 1.75 & 466 & 458 & 1.02 \\
2.22 & 145 & 146 & 1.01 & 1.96 & 382 & 378 & 1.01 \\
2.47 & 119 & 120 & 1.01 & 2.16 & 318 & 315 & 1.01 \\
2.72 & 99 & 100 & 1.01 & 2.36 & 269 & 266 & 1.01 \\
\hline
\end{tabular}

Table 6. FE Results with Different Material Properties F1 for $6 \mathrm{~mm}$ and F2 for $10 \mathrm{~mm}$ (I-section)

\begin{tabular}{ccccccccc}
\hline \multirow{2}{*}{$\begin{array}{c}\text { Buckling } \\
\text { axis }\end{array}$} & \multicolumn{3}{c}{ Austenitic stainless steel } & \multicolumn{4}{c}{ Duplex stainless steel } \\
\cline { 2 - 8 } & $\bar{\lambda}$ & $F_{1}(\mathrm{kN})$ & $F_{2}(\mathrm{kN})$ & $F_{1} / F_{2}$ & $\bar{\lambda}$ & $F_{1}(\mathrm{kN})$ & $F_{2}(\mathrm{kN})$ & $F_{1} / F_{2}$ \\
\hline & 0.39 & 1044 & 1088 & 1.04 & 0.39 & 2257 & 2059 & 1.10 \\
& 0.49 & 976 & 1022 & 1.05 & 0.66 & 1835 & 1735 & 1.06 \\
& 0.59 & 905 & 954 & 1.05 & 0.92 & 1471 & 1372 & 1.07 \\
Major & 0.69 & 832 & 885 & 1.06 & 1.18 & 1115 & 1037 & 1.07 \\
axis & 0.79 & 764 & 815 & 1.07 & 1.45 & 833 & 786 & 1.06 \\
& 0.89 & 701 & 744 & 1.06 & 1.71 & 631 & 601 & 1.05 \\
& 0.99 & 640 & 676 & 1.06 & 1.97 & 491 & 468 & 1.05 \\
& 1.18 & 523 & 548 & 1.05 & 2.24 & 388 & 374 & 1.04 \\
& 1.30 & 474 & 494 & 1.04 & 2.50 & 316 & 304 & 1.04 \\
\hline
\end{tabular}




\begin{tabular}{ccccccccc}
\hline & 1.43 & 429 & 444 & 1.03 & 2.76 & 259 & 252 & 1.03 \\
\hline \multirow{4}{*}{$\begin{array}{c}\text { Minor } \\
\text { axis }\end{array}$} & 0.85 & 682 & 700 & 1.03 & 0.57 & 1926 & 1747 & 1.10 \\
& 1.22 & 467 & 486 & 1.04 & 0.84 & 1539 & 1322 & 1.16 \\
& 1.65 & 332 & 346 & 1.04 & 1.11 & 1173 & 988 & 1.19 \\
& 2.12 & 285 & 296 & 1.04 & 1.37 & 874 & 749 & 1.17 \\
& 2.37 & 214 & 223 & 1.04 & 1.90 & 511 & 458 & 1.11 \\
& 2.62 & 188 & 195 & 1.04 & 2.17 & 405 & 368 & 1.10 \\
& 2.89 & 168 & 172 & 1.03 & 2.57 & 296 & 275 & 1.08 \\
\hline
\end{tabular}

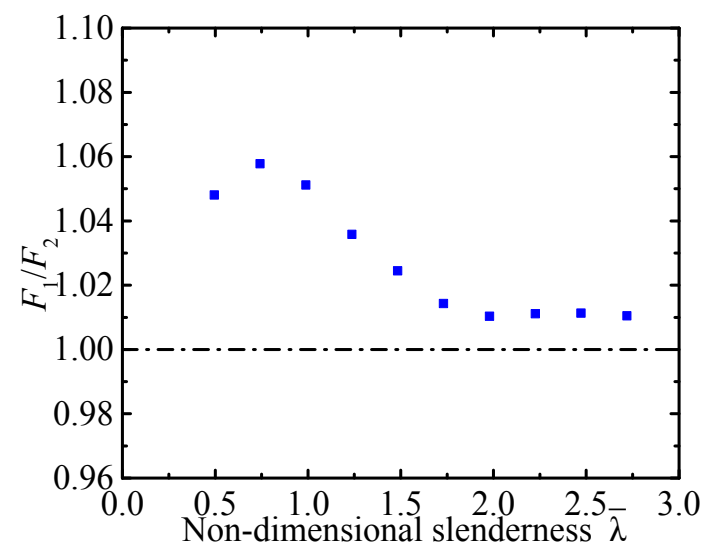

(a) Austenitic box-section

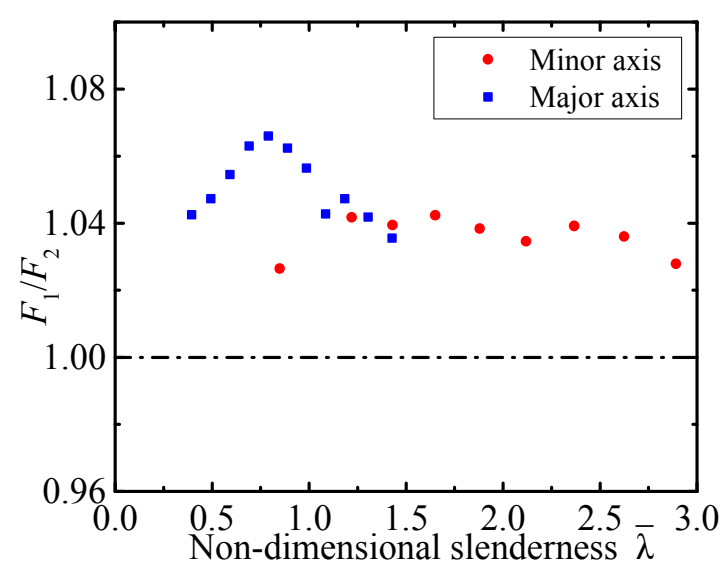

(c) Austenitic I-section

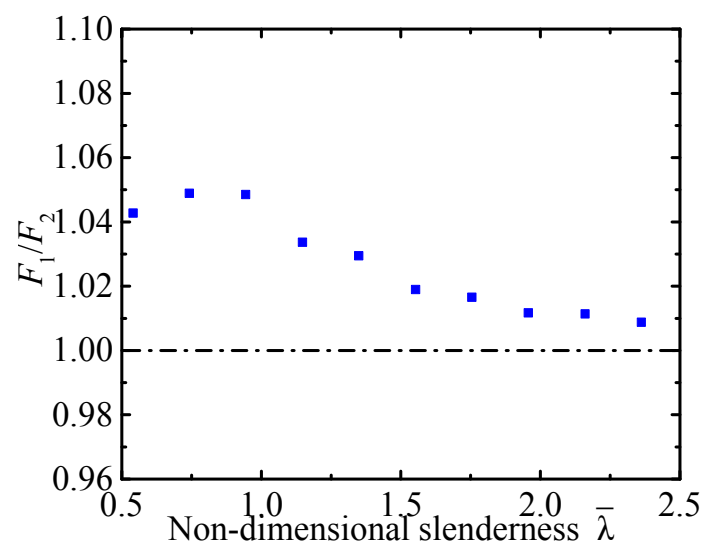

(b) Duplex box-section

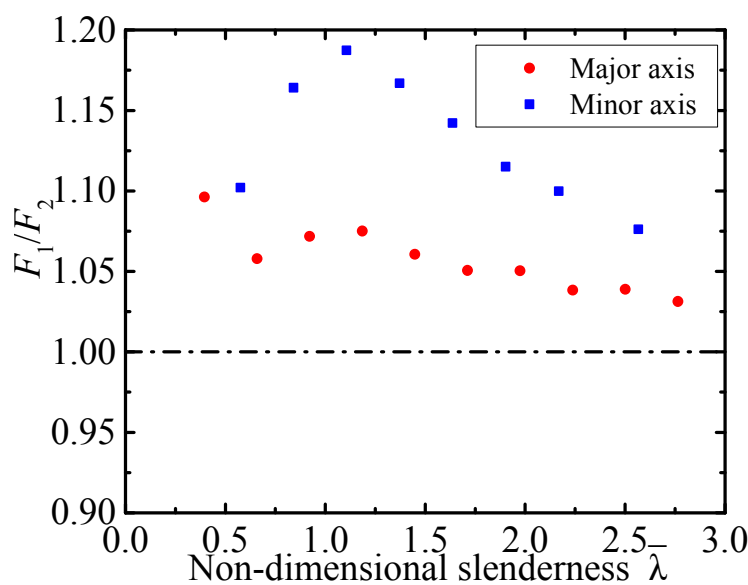

(d) Duplex I-section

Figure 5. The Influence of Material Properties

\subsection{Local width-to-thickness Ratio}

The width-to-thickness ratios ranged from 9 to 40 were adopted for the parametric analysis and the results are shown in Table 7-8. The ultimate load was obtained by numerical analysis, based on which the stability coefficient was calculated. Afterwards the calculated coefficients based on simulation and EN 1993-1-4 are compared and graphically shown in Figure 6, where the stability coefficient $\varphi$ is defined as the ratio of simulated force to normal force $\left(A f_{\mathrm{y}}\right)$. It can be seen from the diagrams that the width-to-thickness ratio barely affects the stability coefficient for both box and I-section members.

Table 7. FE Results with Different Width-to-thickness Ratio (Box-section) 


\begin{tabular}{cccccc}
\hline \multicolumn{3}{c}{ Austenitic stainless steel } & & \multicolumn{3}{c}{ Duplex stainless steel } \\
\hline$B / t$ & $F(\mathrm{kN})$ & $\varphi$ & $B / t$ & $F(\mathrm{kN})$ & $\varphi$ \\
\hline 10 & 168 & 0.46 & 10 & 220 & 0.31 \\
13 & 230 & 0.46 & 13 & 302 & 0.31 \\
17 & 292 & 0.46 & 17 & 384 & 0.31 \\
20 & 355 & 0.46 & 20 & 465 & 0.31 \\
23 & 417 & 0.46 & 23 & 547 & 0.31 \\
27 & 479 & 0.46 & 27 & 629 & 0.31 \\
30 & 542 & 0.46 & 30 & 710 & 0.31 \\
33 & 604 & 0.46 & 33 & 792 & 0.31 \\
37 & 666 & 0.46 & 37 & 874 & 0.31 \\
40 & 728 & 0.46 & 40 & 955 & 0.31 \\
\hline
\end{tabular}

Table 8. FE Results with Different Width-to-thickness Ratio (I-section)

\begin{tabular}{|c|c|c|c|c|c|c|c|}
\hline \multirow[b]{2}{*}{ Buckling form } & \multirow[b]{2}{*}{$\begin{array}{l}\text { Section } \\
\text { position }\end{array}$} & \multicolumn{3}{|c|}{ Austenitic stainless } & \multicolumn{3}{|c|}{ Duplex stainless } \\
\hline & & $B / t$ & $F(\mathrm{kN})$ & $\varphi$ & $B / t$ & $F(\mathrm{kN})$ & $\varphi$ \\
\hline \multirow{3}{*}{ Major axis } & \multirow{3}{*}{ Web } & 15 & 697 & 0.79 & 15 & 1198 & 0.70 \\
\hline & & 20 & 736 & 0.80 & 20 & 1265 & 0.70 \\
\hline & & 25 & 775 & 0.80 & 25 & 1330 & 0.70 \\
\hline \multirow{3}{*}{ Major axis } & \multirow{3}{*}{ Flange } & 15 & 775 & 0.80 & 15 & 1330 & 0.70 \\
\hline & & 18 & 896 & 0.80 & 18 & 1540 & 0.70 \\
\hline & & 21 & 1017 & 0.80 & 21 & 1749 & 0.70 \\
\hline \multirow{5}{*}{ Minor axis } & \multirow{5}{*}{ Web } & 15 & 372 & 0.42 & 15 & 559 & 0.32 \\
\hline & & 20 & 391 & 0.42 & 20 & 587 & 0.32 \\
\hline & & 25 & 411 & 0.42 & 25 & 615 & 0.32 \\
\hline & & 30 & 430 & 0.42 & 30 & 642 & 0.32 \\
\hline & & 35 & 450 & 0.42 & 35 & 670 & 0.32 \\
\hline \multirow{5}{*}{ Minor axis } & \multirow{5}{*}{ Flange } & 9 & 280 & 0.42 & 9 & 416 & 0.32 \\
\hline & & 12 & 345 & 0.42 & 12 & 516 & 0.32 \\
\hline & & 15 & 411 & 0.42 & 15 & 615 & 0.32 \\
\hline & & 18 & 476 & 0.42 & 18 & 714 & 0.32 \\
\hline & & 21 & 542 & 0.42 & 21 & 812 & 0.32 \\
\hline
\end{tabular}
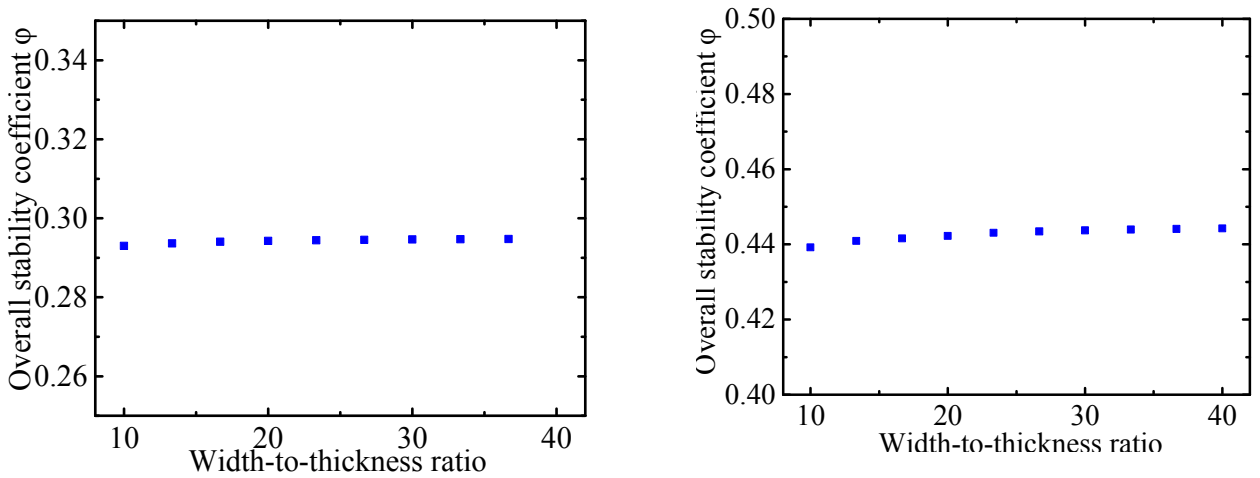
(a) Austenitic box-section

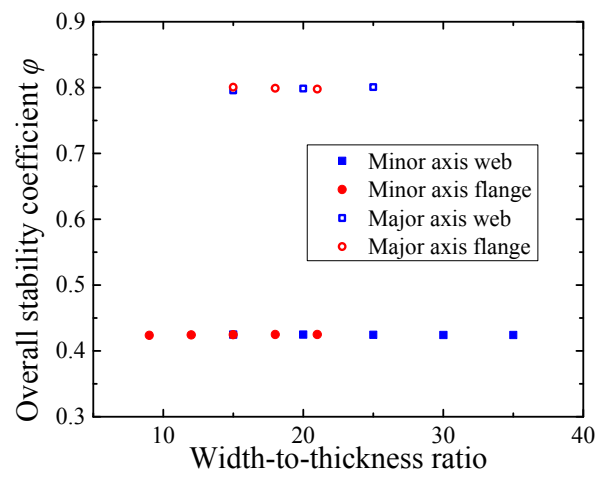

(c) Austenitic I-section (b) Duplex box-section

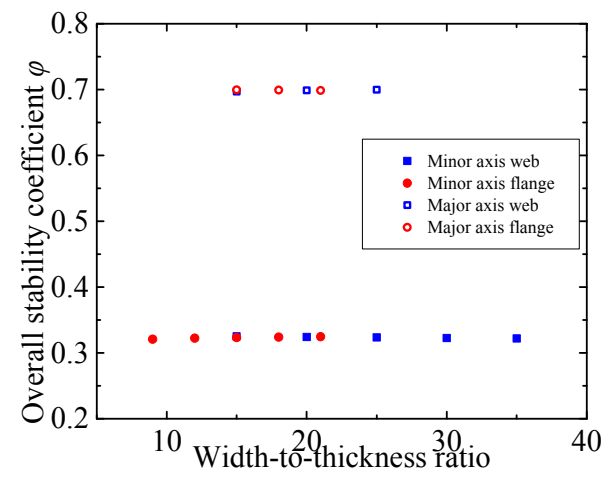

(d) Duplex I-section

Figure 6. The Influence of width-to-thickness ratio

\subsection{Global Slenderness Ratio}

Columns with a wide range of global non-dimensional slenderness ratio have been analyzed using ANSYS and the results are summarized in Table 9-10. The stability coefficients of varying slenderness are depicted in Figure 7. It is obviously illustrated that the coefficient decreases with the increase of non-dimensional slenderness ratio.

Table 9. FE Results with Different Global Non-dimensional Slenderness (Box-section)

\begin{tabular}{cccccc}
\hline \multicolumn{3}{c}{ Austenitic stainless steel } & \multicolumn{3}{c}{ Duplex stainless steel } \\
\hline $\bar{\lambda}$ & $F(\mathrm{kN})$ & $\varphi$ & $\bar{\lambda}$ & $F(\mathrm{kN})$ & $\varphi$ \\
\hline 0.37 & 829 & 0.99 & 0.54 & 1564 & 0.95 \\
0.49 & 775 & 0.92 & 0.64 & 1467 & 0.89 \\
0.62 & 707 & 0.84 & 0.74 & 1362 & 0.83 \\
0.74 & 638 & 0.76 & 0.84 & 1249 & 0.76 \\
0.86 & 571 & 0.68 & 0.94 & 1132 & 0.69 \\
0.99 & 502 & 0.60 & 1.05 & 1017 & 0.62 \\
1.11 & 441 & 0.53 & 1.15 & 907 & 0.55 \\
1.24 & 387 & 0.46 & 1.25 & 808 & 0.49 \\
1.36 & 338 & 0.40 & 1.35 & 722 & 0.44 \\
1.48 & 295 & 0.35 & 1.45 & 645 & 0.39 \\
1.61 & 259 & 0.31 & 1.55 & 575 & 0.35 \\
1.73 & 229 & 0.27 & 1.65 & 518 & 0.31 \\
1.85 & 202 & 0.24 & 1.75 & 466 & 0.28 \\
1.98 & 180 & 0.21 & 1.85 & 421 & 0.25 \\
2.10 & 161 & 0.19 & 1.96 & 382 & 0.23 \\
2.22 & 146 & 0.17 & 2.06 & 348 & 0.21 \\
2.35 & 131 & 0.16 & 2.16 & 318 & 0.19 \\
2.47 & 119 & 0.14 & 2.26 & 292 & 0.18 \\
2.59 & 108 & 0.13 & 2.36 & 269 & 0.16 \\
\hline
\end{tabular}

Table 10. FE Results with Different Global Non-dimensional Slenderness (I-section) 


\begin{tabular}{|c|c|c|c|c|c|c|c|c|c|c|c|c|c|}
\hline \multirow{2}{*}{$\begin{array}{l}\text { Buckling } \\
\text { form }\end{array}$} & \multicolumn{3}{|c|}{$\begin{array}{c}\text { Austenitic stainless } \\
\text { steel }\end{array}$} & \multicolumn{3}{|c|}{$\begin{array}{c}\text { Duplex stainless } \\
\text { steel }\end{array}$} & \multirow{2}{*}{$\begin{array}{l}\text { Buckling } \\
\text { form }\end{array}$} & \multicolumn{3}{|c|}{$\begin{array}{c}\text { Austenitic stainless } \\
\text { steel }\end{array}$} & \multicolumn{3}{|c|}{$\begin{array}{c}\text { Duplex stainless } \\
\text { steel }\end{array}$} \\
\hline & $\bar{z}$ & $\begin{array}{c}F \\
(\mathrm{kN})\end{array}$ & $\varphi$ & $\bar{z}$ & $\begin{array}{c}F \\
(\mathrm{kN})\end{array}$ & $\varphi$ & & $\bar{\lambda}$ & $\begin{array}{c}F \\
(\mathrm{kN})\end{array}$ & $\varphi$ & $\bar{z}$ & $\begin{array}{c}F \\
(\mathrm{kN})\end{array}$ & $\varphi$ \\
\hline \multirow{20}{*}{$\begin{array}{l}\text { Major } \\
\text { axis }\end{array}$} & 0.28 & 1102 & 1.03 & 0.40 & 2257 & 1.01 & \multirow{20}{*}{$\begin{array}{l}\text { Minor } \\
\text { axis }\end{array}$} & 0.40 & 1013 & 1.05 & 0.57 & 1013 & 0.95 \\
\hline & 0.37 & 1044 & 0.98 & 0.51 & 1896 & 1.02 & & 0.49 & 923 & 0.86 & 0.66 & 923 & 0.86 \\
\hline & 0.46 & 976 & 0.92 & 0.61 & 1763 & 0.85 & & 0.59 & 839 & 0.78 & 0.75 & 839 & 0.78 \\
\hline & 0.55 & 905 & 0.85 & 0.72 & 1620 & 0.79 & & 0.68 & 759 & 0.71 & 0.84 & 759 & 0.71 \\
\hline & 0.65 & 832 & 0.78 & 0.83 & 1471 & 0.73 & & 0.77 & 682 & 0.64 & 0.93 & 682 & 0.64 \\
\hline & 0.74 & 764 & 0.71 & 0.93 & 1324 & 0.66 & & 0.87 & 611 & 0.57 & 1.01 & 611 & 0.57 \\
\hline & 0.83 & 701 & 0.65 & 1.04 & 1181 & 0.59 & & 0.96 & 544 & 0.51 & 1.10 & 544 & 0.51 \\
\hline & 0.92 & 640 & 0.59 & 1.15 & 1054 & 0.53 & & 1.05 & 488 & 0.45 & 1.19 & 487 & 0.45 \\
\hline & 1.01 & 580 & 0.54 & 1.25 & 938 & 0.47 & & 1.14 & 437 & 0.41 & 1.28 & 437 & 0.41 \\
\hline & 1.11 & 523 & 0.49 & 1.36 & 833 & 0.42 & & 1.24 & 393 & 0.37 & 1.37 & 393 & 0.37 \\
\hline & 1.20 & 474 & 0.44 & 1.47 & 746 & 0.37 & & 1.33 & 356 & 0.33 & 1.46 & 356 & 0.33 \\
\hline & 1.29 & 429 & 0.40 & 1.58 & 668 & 0.33 & & 1.42 & 323 & 0.30 & 1.54 & 323 & 0.30 \\
\hline & 1.38 & 388 & 0.36 & 1.68 & 601 & 0.30 & & 1.52 & 295 & 0.28 & 1.63 & 295 & 0.28 \\
\hline & 1.48 & 352 & 0.33 & 1.79 & 542 & 0.27 & & 1.61 & 269 & 0.25 & 1.72 & 269 & 0.25 \\
\hline & 1.57 & 320 & 0.30 & 1.90 & 491 & 0.24 & & 1.70 & 247 & 0.23 & 1.81 & 247 & 0.23 \\
\hline & 1.66 & 292 & 0.27 & 2.00 & 446 & 0.22 & & 1.79 & 228 & 0.21 & 1.90 & 228 & 0.21 \\
\hline & 1.75 & 266 & 0.25 & 2.11 & 408 & 0.20 & & 1.89 & 210 & 0.20 & 1.99 & 210 & 0.20 \\
\hline & 1.84 & 243 & 0.23 & 2.22 & 373 & 0.18 & & 1.98 & 194 & 0.18 & 2.07 & 194 & 0.18 \\
\hline & 1.94 & 223 & 0.21 & 2.32 & 343 & 0.17 & & 2.07 & 180 & 0.17 & 2.16 & 180 & 0.17 \\
\hline & 2.12 & 189 & 0.18 & 2.43 & 314 & 0.15 & & 2.17 & 168 & 0.16 & 2.25 & 168 & 0.16 \\
\hline
\end{tabular}

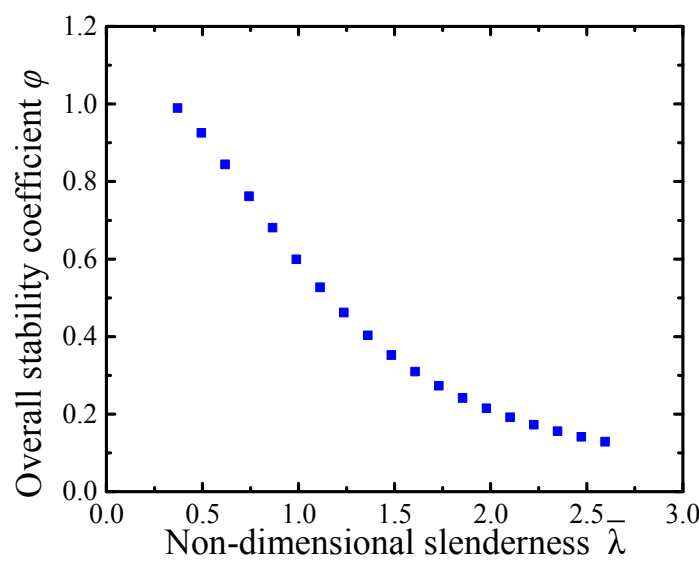

(a) Austenitic box-section

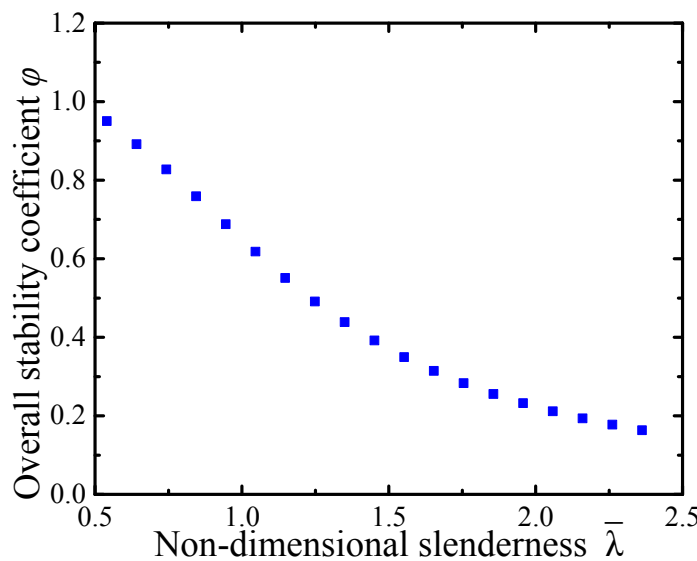

(b) Duplex box-section 


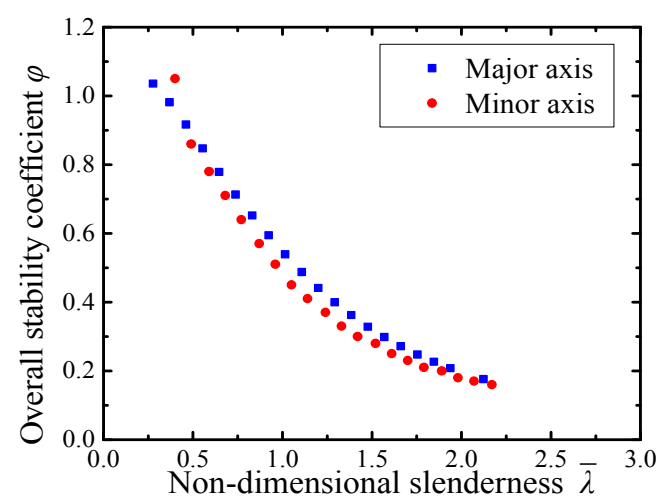

(c) Austenitic I-section

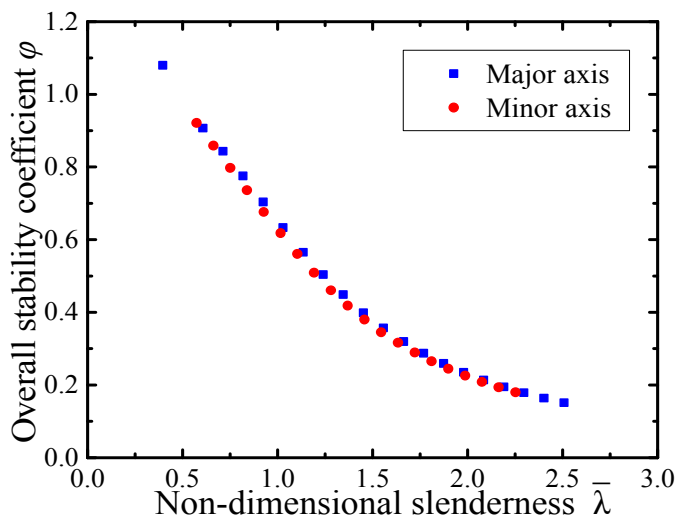

(d) Duplex I-section

Figure 7. The Influence of Non-dimensional Slenderness Ratio

\subsection{Discussion}

It can be concluded that the global non-dimensional slenderness ratio is the most sensitive parameter for the loading capacity of welded stainless steel columns while the effect of the local width-to-thickness ratio can be neglected. The influence of residual stress, initial geometric imperfections and material characteristics is moderate. The results from the parametric analysis identify an appropriate global imperfection level and the necessity of taking residual stress into consideration. These vital parameters would be considered in the new method for flexural design of stainless steel column in the following sections.

\section{DESIGN PROPOSAL OF STAINLESS STEEL WELDED-SECTION COLUMNS}

\subsection{Data from Parametric Analysis}

This section focuses on the investigation on the influence of slenderness on loading capacity of stainless steel columns.

Austenitic and duplex stainless steel columns with $6 \mathrm{~mm}$ and $10 \mathrm{~mm}$ plates are studied. Material properties of the samples are shown in Table 11. Geometric dimensions of stainless steel box-section samples and I-section samples are shown in Table 12 and Table 13 respectively. 
Table 11. Material Properties along the Rolling Direction

\begin{tabular}{ccccccc}
\hline Grade & $t(\mathrm{~mm})$ & $E_{0}(\mathrm{MPa})$ & $f_{\mathrm{y}}(\mathrm{MPa})$ & $f_{\mathrm{u}}(\mathrm{MPa})$ & $\begin{array}{c}\text { Elongation at } \\
\text { fracture (\%) }\end{array}$ & $n$ \\
\hline \multirow{2}{*}{ Austenitic } & 6.00 & 182300 & 282 & 696 & 58.1 & 6.5 \\
& 10.00 & 198700 & 321 & 660 & & 6.0 \\
\multirow{2}{*}{ Duplex } & 6.00 & 191900 & 553 & 798 & 35.0 & 7.0 \\
& 10.20 & 190400 & 547 & 775 & & 6.4 \\
\hline
\end{tabular}

Table 12. Geometric Dimensions of Stainless Steel Box-section Samples

\begin{tabular}{ccccc}
\hline Designation & $B$ & $t$ & $B / t$ & $A$ \\
\hline B1 & 130 & 6 & 21.7 & 2976 \\
B2 & 180 & 10 & 18 & 6800 \\
B3 & 200 & 12 & 16.7 & 9024 \\
B4 & 240 & 16 & 15 & 14336 \\
B5 & 270 & 12 & 22.5 & 12348 \\
B6 & 360 & 16 & 24.6 & 22016 \\
\hline
\end{tabular}

Table 13. Geometric Dimensions of Stainless Steel I-section Samples

\begin{tabular}{cccccccc}
\hline Designation & $H$ & $B$ & $t_{w}$ & $t_{f}$ & $H / t_{w}$ & $B / t_{f}$ & $A$ \\
\hline H1 & 150 & 150 & 6 & 10 & 21.7 & 15 & 3780 \\
H2 & 180 & 150 & 8 & 10 & 20 & 15 & 4280 \\
H3 & 200 & 180 & 10 & 12 & 17.6 & 15 & 6080 \\
H4 & 200 & 200 & 8 & 12 & 22 & 17 & 6208 \\
H5 & 180 & 160 & 12 & 14 & 12.7 & 11.4 & 6304 \\
H6 & 200 & 200 & 14 & 18 & 11.7 & 11.1 & 9496 \\
H7 & 180 & 220 & 16 & 18 & 9 & 12.2 & 10224 \\
\hline
\end{tabular}

In total, 968 columns, including 153 austenitic stainless steel box-section columns, 148 duplex stainless steel box-section columns, 161 austenitic stainless steel I-section columns buckling about minor axis, 176 austenitic stainless steel I-section columns buckling about major axis, 161 duplex stainless steel I-section columns buckling about minor axis and 169 duplex stainless steel I-section columns buckling about major axis were analyzed.

Comparisons between sample data and specification column curves are in Figure 8. It can be observed that section types have no influence on column curves. Besides, when slenderness is greater, calculated curves and EN 1993-1-4 curves fit well while calculated curves and ASCE 8-02 curves fit well for medium-length columns. 


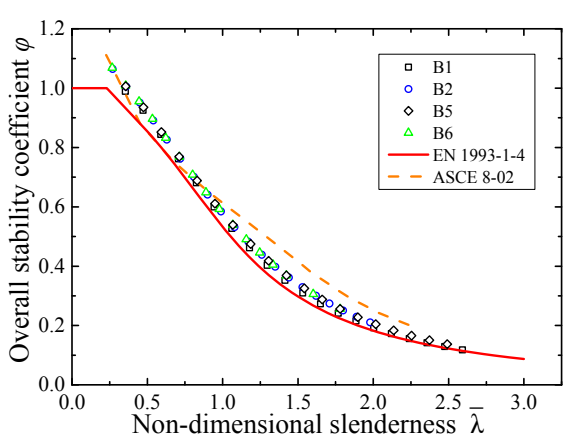

(a) Austenitic box-section

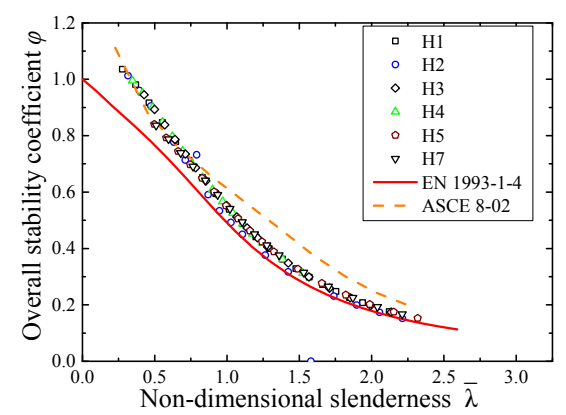

(c) Austenitic I-section (major axis)

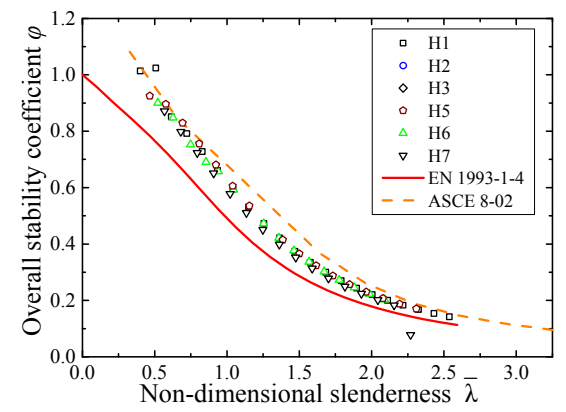

(e) Duplex I-section (major axis)

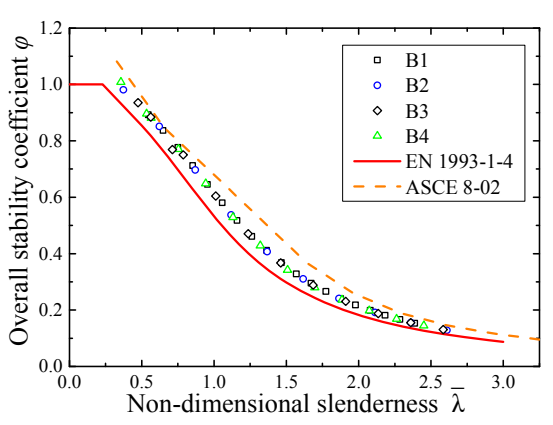

(b) Duplex box-section

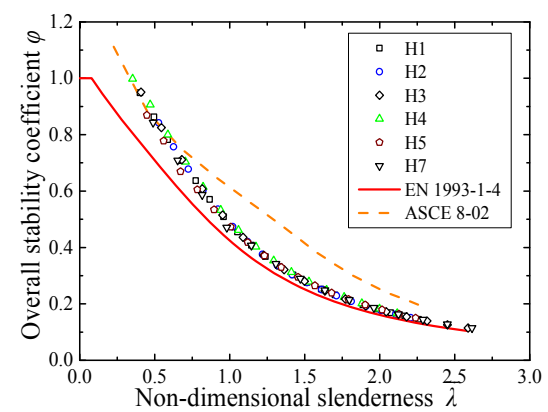

(d) Austenitic I-section (minor axis)

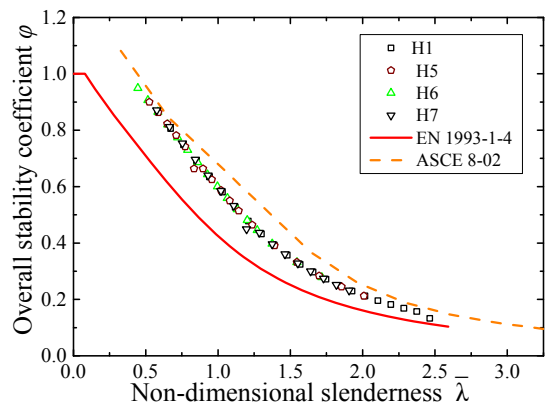

(f) Duplex I-section (minor axis)

Figure 8. Comparisons between Sample Data and Specification Column Curves

\subsection{Proposed Three-segment Model}

EN 1993-1-4 and ASCE 8-02 are commonly used for stainless steel structural design. Considering the limitations of the two design specifications, including conservatism and iteration, a three-segment column curve is proposed based on the test and numerical results. For the 1-segment of the curve, continuous strength method (DSM) proposed by Gardner [18] could be used to capture the beneficial effect of strain-hardening for stocky sections. For the 2-segment, the American cold-formed stainless steel specification could be used considering the nonlinear properties of materials and plastic buckling occurred for intermediate slenderness. For the 3-segment (final segment), Perry formula, based on the edge yield criterion, could be used for large slenderness and the occurred elastic buckling.

Overall stability coefficient formula of welded stainless steel columns can now be expressed as:

$$
\varphi=\left\{\begin{array}{l}
a_{1}+a_{2} * \lambda^{2} \quad \lambda \leq a \\
a_{3} * a_{4}{ }^{\lambda^{2}} \quad a<\lambda \leq b \\
\frac{1}{2 \lambda^{2}}\left[\left(a_{5}+a_{6} \lambda+\lambda^{2}\right)-\sqrt{\left(\left(a_{5}+a_{6} \lambda+\lambda^{2}\right)^{2}-4 \lambda^{2}\right.}\right] \quad b<\lambda
\end{array}\right.
$$


Schematic diagram of this formula is illustrated in Figure 9.

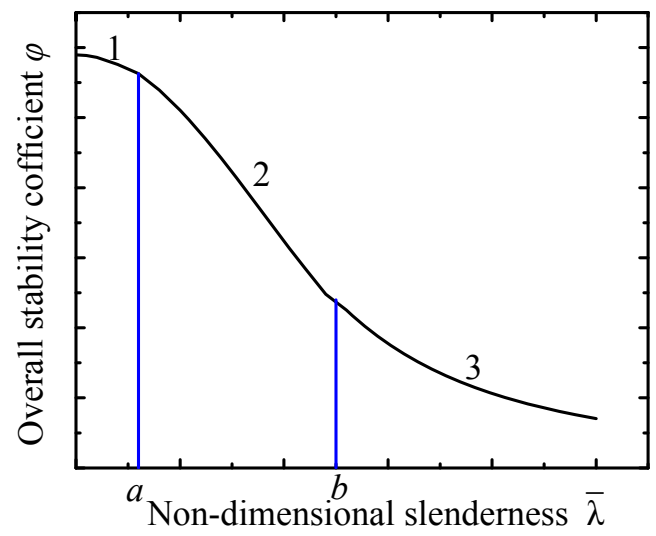

Figure 9. Proposed buckling curves

The first graphical critical point, $a$, can be determined by buckling stress $\sigma$ obtained according to ASCE:

$$
\sigma=\frac{\pi^{2} E_{\mathrm{t}}}{(K L / r)^{2}}=\frac{\pi^{2}}{(K L / r)^{2}} * \frac{E_{\mathrm{O}} f_{\mathrm{y}}}{f_{\mathrm{y}}+0.002 n E_{0}\left(\frac{\sigma}{f_{\mathrm{y}}}\right)^{n-1}}
$$

Section buckling stress is regarded as the critical stress.

The slenderness ratio when $\sigma \gamma_{y}$ is:

$$
(K L / \mathrm{r})^{2}=\frac{\pi^{2} E_{0}}{f_{\mathrm{y}}+0.002 n E_{0}}
$$

The first critical point was evaluated as 0.25 and 0.4 for austenite and duplex stainless steel respectively. The second critical limit, $b$, was determined by curve-fitting, for stainless steel. Values of the second critical limit $b$ and the parameters of $a_{1-a_{6}}$ in Eq. 1 are summarized in Table 14.

\begin{tabular}{|c|c|c|c|c|c|c|c|c|c|}
\hline Material & Section & $\begin{array}{l}\text { Value } \\
\text { type }\end{array}$ & $\begin{array}{c}\text { Second } \\
\text { critical } \\
\text { limit } \\
\end{array}$ & $a_{1}$ & $a_{2}$ & $a_{3}$ & $a_{4}$ & $a_{3}$ & $a_{6}$ \\
\hline \multirow{6}{*}{ Austenitic } & \multirow{2}{*}{ Box-section } & $\begin{array}{l}\text { Fitted } \\
\text { value }\end{array}$ & 1.1 & 1.245 & -2.936 & 1.058 & 0.5428 & 0.8845 & 0.4438 \\
\hline & & $\begin{array}{l}\text { Suggested } \\
\text { value }\end{array}$ & 1.1 & 1.2 & -2.94 & 1.06 & 0.54 & 0.88 & 0.44 \\
\hline & \multirow{2}{*}{$\begin{array}{l}\text { I-section } \\
\text { major axis }\end{array}$} & $\begin{array}{l}\text { Fitted } \\
\text { value }\end{array}$ & 1.3 & 1.111 & -0.9412 & 1.011 & 0.6044 & 0.8983 & 0.3405 \\
\hline & & $\begin{array}{l}\text { Suggested } \\
\text { value }\end{array}$ & 1.3 & 1.1 & -0.94 & 1.01 & 0.6 & 0.9 & 0.34 \\
\hline & \multirow{2}{*}{$\begin{array}{l}\text { I-section } \\
\text { minor axis }\end{array}$} & $\begin{array}{l}\text { Fitted } \\
\text { value }\end{array}$ & 1 & 1.222 & -2.932 & 1.033 & 0.5113 & 0.8494 & 0.5288 \\
\hline & & $\begin{array}{l}\text { Suggested } \\
\text { value }\end{array}$ & 1 & 1.2 & -2.93 & 1.03 & 0.51 & 0.85 & 0.53 \\
\hline \multirow{2}{*}{ Duplex } & \multirow{2}{*}{ Box-section } & $\begin{array}{l}\text { Fitted } \\
\text { value }\end{array}$ & 1.0 & 1.203 & -1.85 & 1.052 & 0.432 & 0.836 & 0.7279 \\
\hline & & Suggested & 1.0 & 1.2 & -1.85 & 1.05 & 0.43 & 0.84 & 0.73 \\
\hline
\end{tabular}

Table 14. Second Critical Limit for Proposed 3-segement Design Curve 


\begin{tabular}{cccccccccc}
\hline \multicolumn{1}{c}{$\begin{array}{c}\text { value } \\
\text { Fitted } \\
\text { I-section } \\
\text { vajor axis }\end{array}$} & 1.1 & 1.106 & 0.7688 & 1.062 & 0.5657 & 1.029 & 0.2247 \\
& $\begin{array}{c}\text { Suggested } \\
\text { value } \\
\text { Fitted }\end{array}$ & 1.1 & 1.1 & -0.77 & 1.06 & 0.57 & 1.03 & 0.23 \\
$\begin{array}{c}\text { I-section } \\
\text { value } \\
\text { minor axis }\end{array}$ & $\begin{array}{c}\text { Suggested } \\
\text { value }\end{array}$ & 1.2 & 1.088 & -1.044 & 0.9964 & 0.5426 & 1.039 & 0.3772 \\
\hline
\end{tabular}

\subsection{Reliability Analysis}

Each parameter of the proposed equation has been fitted using MATLAB. In order to verify the accuracy of the design method, the ratio of FE stability coefficient $\varphi_{1}$ to calculated stability coefficient $\varphi_{2}$ is illustrated in Figure 10. In Figure 9, $\varphi_{1} / \varphi_{2}$ fluctuates between 0.95 and 1.05, which shows the reliability of the design proposal.

The proposed design curve and the design curves from EN1993-1-4 and ASCE8-02 are compared in Figure 11. It can be observed that the proposed column curve is in-between the EN 1993-1-4 and the ASCE 8-02 curves. It also indicates that the discrepancies between the calculated overall stability coefficient and the EN 1993-1-4 curve for austenitic stainless steel member are smaller than that of duplex stainless steel. It indicates that different material grades significantly impact the overall stability loading capacity and therefore separated design curves are needed. Also the discrepancies of the major-axis buckling are less than that of the minor-axis buckling in terms of I-section members and thus separated design curves are needed. It can also be seen that the EN 1993-1-4 produced an evidently conservative design for welded stainless steel columns. The design loading capacity to test and simulated data ratio are approximately 1 for both box-section and I-section members, verifying the rationality of the proposed formula. The mean value, COV and reliability index of tests and FEA to the design predictions ratio are shown in Tables 15-17. The mean value of the experimental and numerical axial strengths over the modified design strengths is nearer to 1.0, while the COV of which is much lower. And the reliability index of the proposed method is generally higher than other provisions. In general, the test and numerical results on welded stainless steel columns can be accurately predicted by the proposed column curves. It should also be noted that the modified design proposal does not require iterative process in calculating the column strength as shown in ASCE's provisions.

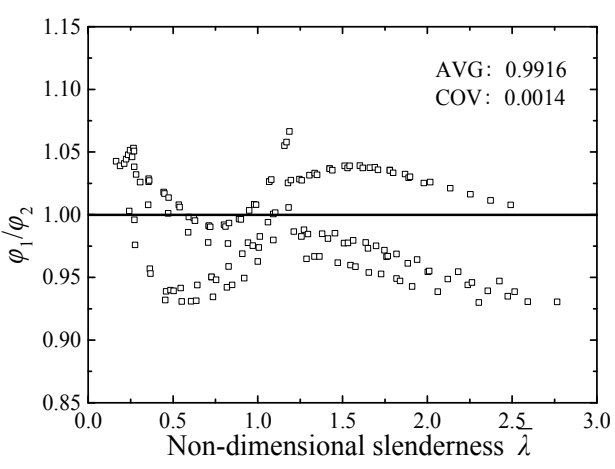

(a) Austenitic box-section

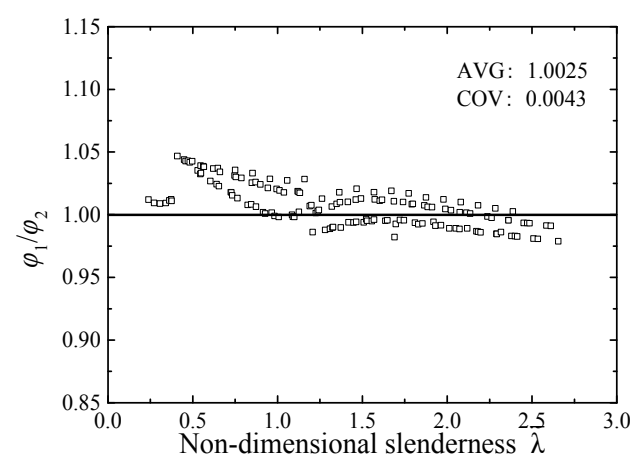

(b) Duplex box-section 


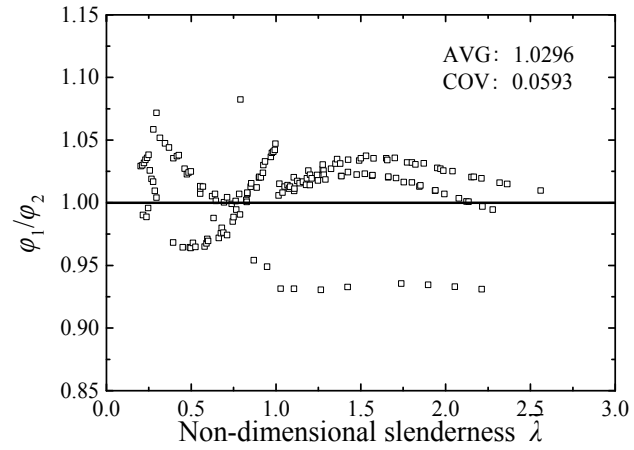

(c) Austenitic I-section (major axis)

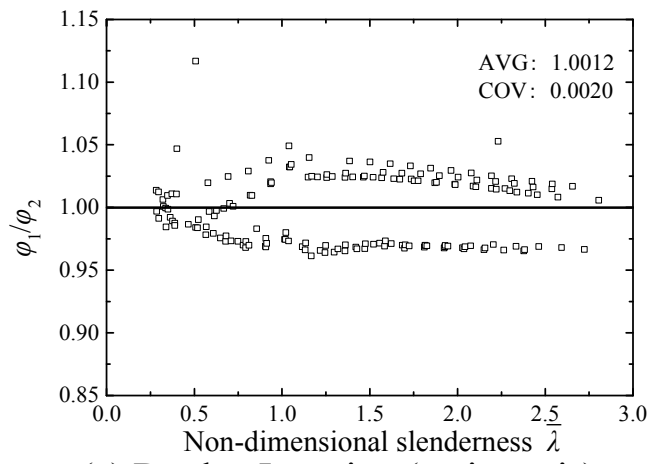

(e) Duplex I-section (major axis)

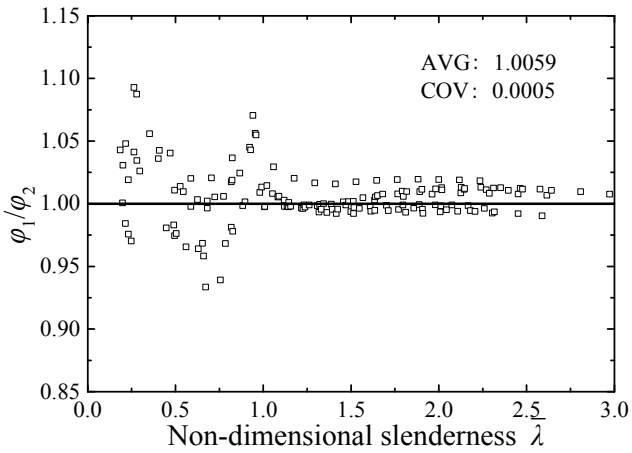

(d) Austenitic I-section (minor axis)

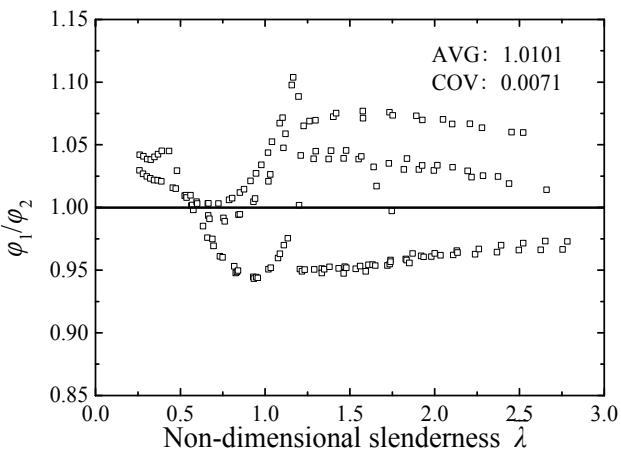

(f) Duplex I-section (minor axis)

Figure 10. Comparison of FE and Calculated Stability Coefficient

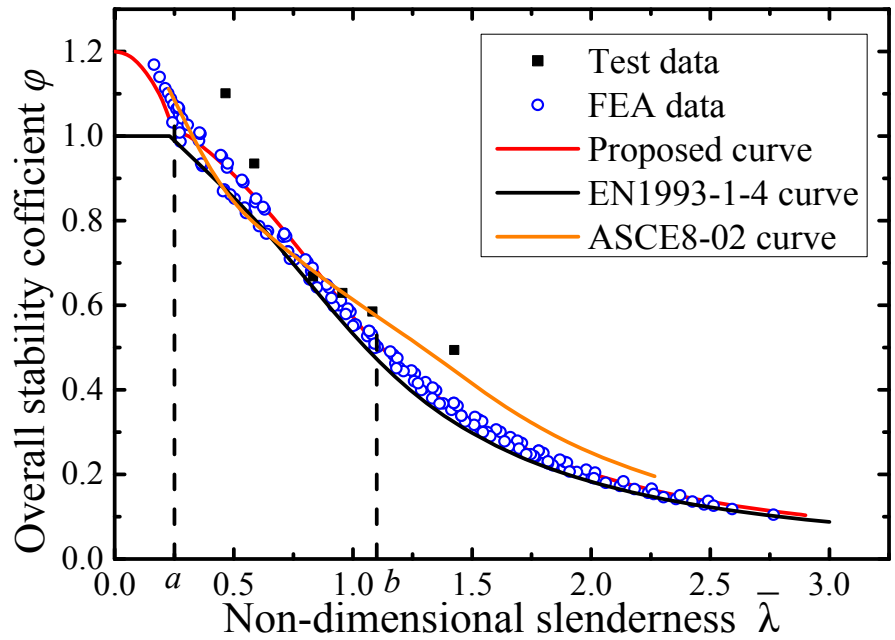

(a) Austenitic box-section 


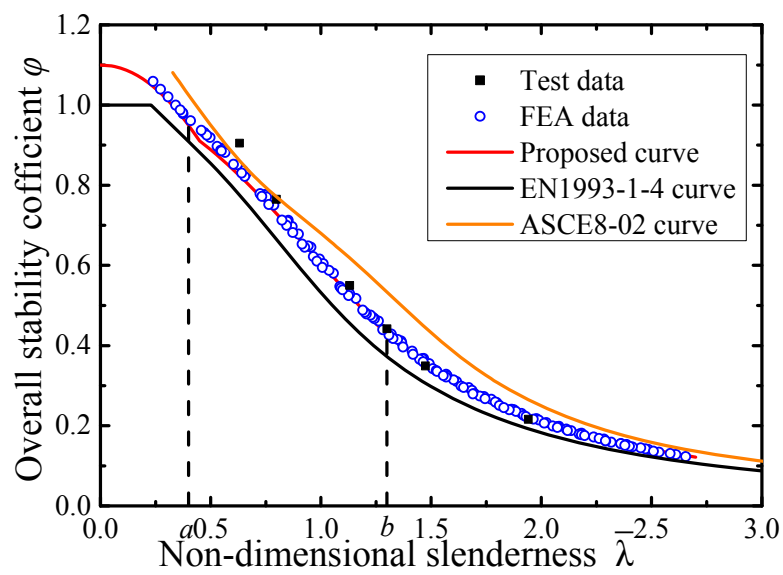

(b) Duplex box-section

Table 15. Comparison of Test and FEA Results with Design Predictions (Box-section)

\begin{tabular}{|c|c|c|c|c|c|c|}
\hline \multirow{4}{*}{ Parameters } & \multicolumn{3}{|c|}{ Austenitic stainless steel } & \multicolumn{3}{|c|}{ Duplex stainless steel } \\
\hline & \multicolumn{2}{|c|}{ Current design rules } & \multirow{2}{*}{$\begin{array}{c}\text { Modified } \\
\text { design rules }\end{array}$} & \multicolumn{2}{|c|}{ Current design rules } & \multirow{2}{*}{$\begin{array}{c}\text { Modified } \\
\text { design rules } \\
P_{\mathrm{mg}} \text { and } P_{\mathrm{FE}}\end{array}$} \\
\hline & $P_{\mathrm{tw}}$ and $P_{\mathrm{FE}}$ & $P_{\mathrm{ANR}}$ and $P_{\mathrm{FE}}$ & & $P_{\mathrm{arg}}$ and $P_{\mathrm{FE}}$ & $P_{\mathrm{apg}}$ and $P_{\mathrm{FE}}$ & \\
\hline & $P_{\mathrm{ECO}}$ & $P_{\text {AGCE }}$ & $P$ P.MO & $P_{\mathrm{EC} 3}$ & $P_{\text {AGEL }}$ & $P_{\text {MO }}$ \\
\hline $\begin{array}{c}\text { Number of } \\
\text { data }\end{array}$ & 159 & 12 & 159 & 152 & 12 & 152 \\
\hline AVG & 0.9911 & 1.3574 & 0.9962 & 0.9816 & 1.3402 & 1.0012 \\
\hline $\mathrm{COV}$ & 0.0039 & 0.0402 & 0.0026 & 0.0045 & 0.0414 & 0.0025 \\
\hline $\begin{array}{l}\text { Reliability } \\
\text { index }\end{array}$ & 15.89 & 6.48 & 19.30 & 7.87 & 6.31 & 20.53 \\
\hline
\end{tabular}

Table 16. Comparison of Test and FEA Results with Design Predictions (I-section buckling around major axis)

\begin{tabular}{|c|c|c|c|c|c|c|}
\hline \multirow{4}{*}{ Parameters } & \multicolumn{3}{|c|}{ Austenitic stainless steel } & \multicolumn{3}{|c|}{ Duplex stainless steel } \\
\hline & \multicolumn{2}{|c|}{ Current design rules } & \multirow{2}{*}{$\begin{array}{c}\text { Modified } \\
\text { design rules } \\
P_{\text {ew }} \text { and } P_{\text {FE }}\end{array}$} & \multicolumn{2}{|c|}{ Current design rules } & \multirow{2}{*}{$\begin{array}{c}\text { Modified } \\
\text { design rules } \\
P_{\mathrm{eng}} \text { and } P_{\mathrm{FE}}\end{array}$} \\
\hline & $P_{\mathrm{ewg}}$ and $P_{\mathrm{Fg}}$ & $P_{\mathrm{twg}}$ and $P_{\mathrm{FE}}$ & & $P_{\mathrm{ewg}}$ and $P_{\mathrm{FE}}$ & $P_{\mathrm{awg}}$ and $P_{\mathrm{g}}$ & \\
\hline & $P_{\mathrm{EQ3}}$ & $P_{\mathrm{ASCH}}$ & PMO & $P_{\mathrm{BG}}$ & $P_{\mathrm{ASOR}}$ & Pun \\
\hline $\begin{array}{c}\text { Number of } \\
\text { data }\end{array}$ & 167 & 22 & 167 & 172 & 21 & 172 \\
\hline $\mathrm{AVG}$ & 1.1051 & 1.2815 & 0.9987 & 1.0520 & 1.0933 & 0.9998 \\
\hline $\mathrm{COV}$ & 0.1057 & 0.0864 & 0.2189 & 0.0032 & 0.0028 & 0.0009 \\
\hline $\begin{array}{l}\text { Reliability } \\
\text { index }\end{array}$ & 3.24 & 12.28 & 12.06 & 18.57 & 19.45 & 33.38 \\
\hline
\end{tabular}


Table 17. Comparison of Test and FEA Results with Design Predictions (I-section buckling around minor axis)

\begin{tabular}{|c|c|c|c|c|c|c|}
\hline \multirow{4}{*}{ Parameters } & \multicolumn{3}{|c|}{ Austenitic stainless steel } & \multicolumn{3}{|c|}{ Duplex stainless steel } \\
\hline & \multicolumn{2}{|c|}{ Current design rules } & \multirow{2}{*}{$\begin{array}{c}\text { Modified } \\
\text { design rules }\end{array}$} & \multicolumn{2}{|c|}{ Current design rules } & \multirow{2}{*}{$\begin{array}{c}\text { Modified } \\
\text { design rules } \\
P_{\mathrm{eng}} \text { and } P_{\mathrm{FE}}\end{array}$} \\
\hline & $P_{\mathrm{ewp}}$ and $P_{\mathrm{FE}}$ & $P_{\mathrm{tw}}$ and $P_{\mathrm{FE}}$ & & $P_{\mathrm{ewp}}$ and $P_{\mathrm{FE}}$ & $P_{\mathrm{axp}}$ and $P_{\mathrm{gE}}$ & \\
\hline & $P_{\mathrm{EC} 3}$ & $P_{\mathrm{ABCE}}$ & $8 \times 10$ & $P_{\mathrm{HC}}$ & $P_{\mathrm{ABCE}}$ & $P_{10}$ \\
\hline $\begin{array}{c}\text { Number of } \\
\text { data }\end{array}$ & 167 & 22 & 167 & 172 & 21 & 172 \\
\hline AVG & 1.0527 & 1.1841 & 0.9905 & 1.2227 & 1.0896 & 1.0824 \\
\hline $\mathrm{COV}$ & 0.0046 & 0.0312 & 0.0019 & 0.0037 & 0.0115 & 0.0022 \\
\hline $\begin{array}{l}\text { Reliability } \\
\text { index }\end{array}$ & 14.59 & 6.42 & 12.85 & 20.02 & 9.73 & 23.00 \\
\hline
\end{tabular}

\section{CONCLUSIONS}

This paper has presented an extensive parametric investigation with an aim to proposing a new design method for flexural design of fabricated austenitic and duplex stainless steel columns. Complementary experimental programme were conducted and the corresponding finite element modelling methodologies were validated in early investigations [20, 21]. The key parameters, including initial global geometric imperfections, residual stress, local width-to-thickness ratio, material properties and non-dimensional slenderness ratio were assessed. A total of 301 welded box-section and 667 welded I-section columns were simulated, which were used to evaluate the current design provisions and the calculation method proposed in this paper. The design curves provided in EN 1993-1-4 were confirmed to give conservative predictions while ASCE 8-02 give slightly overestimated predictions.

Based on the complementary tests and simulated results, a three-segment design curve was proposed for the flexural design of fabricated austenitic and duplex stainless steel columns and demonstrated reliable predictions.

\section{ACKNOWLEDGEMENT}

This study is supported by the National Natural Science Foundation of China (Grant NO. 51478019) and Beijing NOVA programme 2016.

\section{REFERENCES}

[1] Baddoo, N. R., "Stainless Steel in Construction: A Review of Research, Applications, Challenges and Opportunities", Journal of Constructional Steel Research, 2008, Vol. 64, No. 11, pp. 1199-1206.

[2] EN 1993-1-4, Eurocode 3: "Design of Steel Structures Part 1-4: General RulesSupplementary Rules for Stainless Steels", Brussels: European Committee for Standardization, 2006.

[3] SEI/ASCE 8-02, "Specification for the Design of Cold-Formed Stainless Steel Structural Members, Virginia: American Society of Civil Engineers”, 2002.

[4] CECS 410:2015, “Technical Code of Stainless Steel Structures", Nanjing: Ministry of Industry and Information Technology of the People's Republic of China, 2015. 
[5] Mirambell, E. and Real, E., "On the Calculation of Deflections in Structural Stainless Steel Beams: An Experimental and Numerical Investigation", Journal of Constructional Steel Research, 2000, Vol. 54, No. 1, pp. 109-133.

[6] Quach, W. M., Teng, J. G. and Chung, K. F., "Three-stage Full-range Stress-strain Model for Stainless Steels", Journal of Structural Engineering, 2008, Vol. 134, No. 9, pp. 1518-1527.

[7] Gardner, L. and Cruise, R. B., "Modeling of Residual Stresses in Structural Stainless Steel Sections", Journal of Structural Engineering, 2009, Vol. 135, No. 1, pp. $42-53$.

[8] Quach, W. M., Teng, J. G. and Chung, K. F., "Residual Stresses in Press-braked Stainless Steel Sections, I: Coiling and Uncoiling of Sheets", Journal of Constructional Steel Research, 2009, Vol. 65, No. 8, pp. 1803-1815.

[9] Yuan, H. X., Wang, Y. Q., Shi, Y. J. and Gardner, L., "Residual Stress Distributions in Welded Stainless Steel Sections”, Thin-Walled Structures, 2014, Vol. 79, pp. 38-51.

[10] Wang, Y. Q., Yang, L., Gao, B., Shi, Y. J. and Yuan, H. X., "Experimental Study of Lateral-torsional Buckling Behaviour of Stainless Steel Welded I-section Beams", International Journal of Steel Structures, 2014, Vol. 14, No. 2, pp. 411-420.

[11] Yang, L., Wang, Y. Q., Gao, B., Shi, Y. J. and Yuan, H. X., "Two Calculation Methods for Buckling Reduction Factors of Stainless Steel Welded I-section Beams", Thin-Walled Structures, 2014, Vol. 83, pp. 128-136.

[12] Yuan, H. X., Wang, Y. Q., Gardner, L. and Shi, Y. J., "Local-overall Interactive Buckling of Welded Stainless Steel Box Section Compression Members”, Engineering Structures, 2014, Vol. 67, No. 4, pp. 62-76.

[13] Shu, G., Zheng, B. and Xin, L., "A New Design Method for Stainless Steel Columns Subjected to Flexural Buckling", Thin-Walled Structures, 2014, Vol. 83, pp. 43-51.

[14] Shu, G., Zheng, B. and Shen, X., "Experimental and Theoretical Study on the Behaviour of Cold-formed Stainless Steel Stub Columns", International Journal of Steel Structures, 2013, Vol. 13, No. 1, pp. 141-153.

[15] Huang, Y. and Young, B., "Design of Cold-formed Lean Duplex Stainless Steel Members in Combined Compression and Bending", Journal of Structural Engineering, 2014, Vol. 141, No. 5, pp. 04014138.

[16] Gardner, L. and Nethercot, D. A., "Experiments on Stainless Steel Hollow Sections-Part 1: Material and Cross-sectional Behaviour", Journal of Constructional Steel Research, 2004, Vol. 60, No. 9, pp. 1291-1318.

[17] Gardner, L. and Nethercot, D. A., "Experiments on Stainless Steel Hollow Sections - Part 2: Member Behaviour of Columns and Beams", Journal of Constructional Steel Research, 2004, Vol. 60, No. 9, pp. 1319-1332.

[18] Gardner, L., "The Continuous Strength Method", Proceedings of the Institution of Civil Engineers-structures and Buildings, 2008, Vol. 161, No. 3, pp. 127-133.

[19] AS/NZS 4673: "Cold- formed Stainless Steel Structures", Sydney: Joint Technical Committee, 2001.

[20] Yang, L., Zhao, M. H., Xu, D., Shang, F., Yuan, H. X., Wang, Y. Q. and Zhang, Y., "Flexural Buckling Behaviour of Welded Stainless Steel Box-section Columns", Thin-Walled Structures, 2016, Vol. 104, pp. 185-197.

[21] Yang, L., Zhao, M. H., Chan, T. M., Shang, F. and Xu, D. C., "Flexural Buckling of Welded Austenitic and Duplex Stainless Steel I-section Columns", Journal of Constructional Steel Research, 2016, Vol. 122, pp. 339-353. 


\section{NOTATION}

The following symbols are used in this paper:

$A=$ the section area;

$B=$ the width of the member section;

$E_{0}=$ the initial elasticity modulus;

$F_{\mathrm{y}}=$ bearing capacity;

$F_{\mathrm{w}}=$ bearing capacity with residual stress considered;

$F_{0.001}=$ the ultimate load of the members with $0.001 L$ geometric imperfection;

$F_{0.002}=$ the ultimate load of the members with $0.002 L$ geometric imperfection;

$F_{0.0005}=$ the ultimate load of the members with $0.005 \mathrm{~L}$ geometric imperfection;

$f_{\mathrm{y}}=$ the $0.2 \%$ proof stress;

$H=$ the height of the member section;

$P_{\mathrm{EC} 3}=$ the ultimate buckling load calculated according to EN1993-1-4;

$P_{\text {ASCE }}=$ the ultimate buckling load calculated according to ASCE-8-02;

$P_{\exp }=$ the tested ultimate buckling load;

$P_{\mathrm{FE}}=$ the simulated ultimate load with the residual stress considered;

$n=$ the strain-hardening exponent;

$t=$ the thickness of the member section;

$t_{\mathrm{f}}=$ the thickness of the flange;

$t_{\mathrm{w}}=$ the thickness of the web;

$\delta_{0}=$ the initial geometric imperfection;

$\bar{\lambda}=$ the non-dimensional slenderness ratio;

$\sigma=$ buckling stress;

$\varphi=$ overall stability coefficient. 